\title{
GENERALIZED MAXIMAL FUNCTIONS AND RELATED OPERATORS ON WEIGHTED MUSIELAK-ORLICZ SPACES
}

\author{
Ana Bernardis, Estefanía Dalmasso and Gladis Pradolini \\ Facultad de Ingeniería Química, Universidad Nacional del Litoral (UNL) \\ and Instituto de Matemática Aplicada del Litoral, CONICET - UNL \\ Güemes 3450, 3000 Santa Fe, Argentina; bernard@santafe-conicet.gov.ar \\ Facultad de Ingeniería Química, Universidad Nacional del Litoral (UNL) \\ and Instituto de Matemática Aplicada del Litoral, CONICET - UNL \\ Güemes 3450, 3000 Santa Fe, Argentina; edalmasso@santafe-conicet.gov.ar \\ Facultad de Ingeniería Química, Universidad Nacional del Litoral (UNL) \\ and Instituto de Matemática Aplicada del Litoral, CONICET - UNL \\ Güemes 3450, 3000 Santa Fe, Argentina; gpradolini@santafe-conicet.gov.ar
}

\begin{abstract}
We characterize the class of weights related to the boundedness of maximal operators associated to a Young function $\eta$ in the context of variable Lebesgue spaces. Fractional versions of these results are also obtained by means of a weighted Hedberg type inequality. These results are new even in the classical Lebesgue spaces. We also deal with Wiener's type inequalities for the mentioned operators in the variable context. As applications of the strong type results for the maximal operators, we derive weighted boundedness properties for a large class of operators controlled by them.
\end{abstract}

\section{Introduction and preliminaries}

The variable exponent Lebesgue spaces arise when we deal with a great number of applications in partial differential equations. In fact, they seem to be the natural context in order to describe the behaviour of certain classes of fluids, called electrorheological fluids, which have the ability to significantly modify its mechanical properties when an electric field is applied (see for example [47]). Other applications that find in these spaces an adequate development framework for their theory are the processes of image restoration [6] and partial differential equations (see for instance [1] and [24]).

The boundedness of many operators in harmonic analysis that appear in connection with the study of regularity properties of the solutions of partial differential equations were widely considered in the variable context by different authors, see for instance [9], [11], [14], [15], [16], [30], [31], [32], [38], [39] and [40] for the HardyLittlewood maximal function $M,[5],[21],[22]$ and [28] for the fractional maximal function $M_{\alpha}$, [18] and [33] for Calderón-Zygmund operators and their commutators, and [1], [10], [24] and [28] for potential type operators (see [13] for other classical operators).

doi:10.5186/aasfm.2014.3904

2010 Mathematics Subject Classification: Primary 42B25.

Key words: Musielak-Orlicz spaces, weights, maximal functions.

The authors were suportted by Consejo Nacional de Investigaciones Científicas y Técnicas and Universidad Nacional del Litoral. 
It is well known that many of the operators mentioned above are controlled, in some sense, by maximal operators. Sometimes this control is given in the norm of the spaces where they act. A typical inequality that describes this fact on weighted Lebesgue spaces is the following

$$
\int_{\mathbf{R}^{n}}|\mathcal{T} f(x)|^{p} w(x) d x \leq C \int_{\mathbf{R}^{n}}\left|\mathcal{M}_{\mathcal{T}} f(x)\right|^{p} w(x) d x, \quad 0<p<\infty
$$

where $w$ is a weight, $\mathcal{T}$ is certain integral operator and $\mathcal{M}_{\mathcal{T}}$ is the maximal operator referred to above. Thus, it is clear that the operator $\mathcal{T}$ inherits the continuity properties of $\mathcal{M}_{\mathcal{T}}$ in the corresponding space. For example, if $\mathcal{T}$ is a Calderón-Zygmund operator, (1.1) holds by taking $\mathcal{M}_{\mathcal{T}}=M$. This result was proved by Coifman in [7] in the unweighted context and by Coifman and Fefferman in [8] for $w \in A_{\infty}$. When $\mathcal{T}$ is the fractional integral operator, then (1.1) holds with $\mathcal{M}_{\mathcal{T}}=M_{\alpha}$ by taking $w \in A_{\infty}$ (see [36]). On the other hand, when we deal with commutators of singular or fractional integrals in inequalities in the spirit of (1.1) the maximals involved are iterations of the Hardy-Littlewood maximal function and compositions of them with fractional maximals, respectively (see for example [2], [19], [42], [43]). It is well known that this type of operators are equivalent to certain maximal operators associated to a Young function of $L \log L$ type (see for example [2], [3], [42], [43]). For more general Young functions, the maximal operators involved control operators of convolution type with kernels satisfying certain Hörmander conditions associated to the Young functions that define the maximals (see [34] and [35], and [4] for the fractional case).

Inequalities of Fefferman-Stein type were also considered by many authors (see [2], [3], [27], [34], [41], [44]).

In this article, we are interested in the study of the continuity properties of the maximal operator mentioned above, that is, a maximal associated to a Young function $\eta, M_{\eta}$, in the context of variable Lebesgue spaces with weights. We first characterize the weights involved in the case that the Young function defining the maximal operator is of $L \log L$ type and the weights obtained are of the type defined in [9]. In that article, the authors succeed in giving the class of weights that characterizes the boundedness of $M$ in $L_{w}^{p(\cdot)}\left(\mathbf{R}^{n}\right)$ (see below for the definition of this space). When $\eta$ satisfies certain Dini type condition we obtain a sufficient result for the boundedness of $M_{\eta}$ in $L_{w}^{p(\cdot)}\left(\mathbf{R}^{n}\right)$. As we said before, these results allow us to derive weighted estimates in $L_{w}^{p(\cdot)}\left(\mathbf{R}^{n}\right)$ for a large class of operators controlled by $M_{\eta}$. We show that this class contains, for example, singular and fractional integral operators with kernels satisfying certain Hörmander type condition and their commutators, which have, as particular cases, Calderón-Zygmund and fractional integral operators.

Another type of inequality we shall deal with are generalizations of Wiener's inequality in the variable context. In this way, the main results were obtained by Cruz-Uribe and Fiorenza in [11], where they proved that if $B$ is any ball and $\epsilon>0$, the inequality

$$
\int_{B} M f(x) d x \leq 2|B|+C \int_{\mathbf{R}^{n}}|f(x)|^{p(x)} \log (e+|f(x)|)^{q(x)} d x
$$


holds for every bounded exponent $p: \mathbf{R}^{n} \rightarrow[1, \infty)$, where $q(x)=\max \left\{\epsilon^{-1}(\epsilon+1-\right.$ $p(x)), 0\}$. Note that when $p=1$ then $q=1$ and (1.2) is Wiener's inequality. By requiring additional properties on the exponent, they improved their local integrability results.

Since the maximal operator $M_{\eta}$ is greater than a constant times $M$, it seems to be interesting to study the local integrability of this maximal operator. Thus, in this direction we obtain Wiener's type results by following the ideas in [11].

On the other hand, fractional versions of all of the results described above were obtained. A useful tool in order to have the fractional case is a weighted pointwise control inequality between the fractional maximal operator $M_{\alpha, \eta}$ and $M_{\psi}$ where $\eta$ and $\psi$ are certain Young functions. This type of inequality is an extension of Hedberg's inequality (see [25]). Another extensions can be found in [5], [21] and [22].

We now introduce some notation and preliminary results.

Let $p: \mathbf{R}^{n} \rightarrow[1, \infty)$ be a measurable function. Given a measurable set $E \subset \mathbf{R}^{n}$,

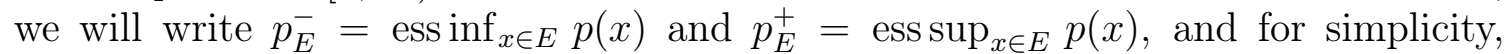
$p_{\mathbf{R}^{n}}^{-}=p^{-}$and $p_{\mathbf{R}^{n}}^{+}=p^{+}$. By $\mathcal{P}\left(\mathbf{R}^{n}\right)$ we will denote the set of measurable functions $p: \mathbf{R}^{n} \rightarrow[1, \infty)$ and by $\mathcal{P}^{*}\left(\mathbf{R}^{n}\right)$ the set of $p \in \mathcal{P}\left(\mathbf{R}^{n}\right)$ such that $p^{+}<\infty$.

Given $p \in \mathcal{P}^{*}\left(\mathbf{R}^{n}\right)$, we say that a measurable function $f$ belongs to $L^{p(\cdot)}\left(\mathbf{R}^{n}\right)$ if for some $\lambda>0$

$$
\int_{\mathbf{R}^{n}}\left(\frac{|f(x)|}{\lambda}\right)^{p(x)} d x<\infty .
$$

In this case, we define the Luxemburg norm in $L^{p(\cdot)}\left(\mathbf{R}^{n}\right)$ by

$$
\|f\|_{p(\cdot)}=\inf \left\{\lambda>0: \int_{\mathbf{R}^{n}}\left(\frac{|f(x)|}{\lambda}\right)^{p(x)} d x \leq 1\right\} .
$$

For more information about $L^{p(\cdot)}$ spaces, see [12], [17] and [29].

It is well known that $\left(L^{p(\cdot)}\left(\mathbf{R}^{n}\right),\|\cdot\|_{p(\cdot)}\right)$ is a Banach space. This space is a particular case of Musielak-Orlicz space by taking $\Phi(x, t)=t^{p(x)}$ (for more information about Musielak-Orlicz spaces, see [16] or [37]).

The following conditions on the exponent arise related with the boundedness of the Hardy-Littlewood maximal operator (see, for example, [14] or [15]). We will say that $p \in \mathcal{P}^{\log }\left(\mathbf{R}^{n}\right)$ if $p \in \mathcal{P}^{*}\left(\mathbf{R}^{n}\right)$ and if it satisfies the following inequalities

$$
|p(x)-p(y)| \leq \frac{C}{\log (e+1 /|x-y|)} \quad \forall x, y \in \mathbf{R}^{n},
$$

and

$$
|p(x)-p(y)| \leq \frac{C}{\log (e+|x|)} \quad \forall|y| \geq|x| .
$$

Conditions (1.3) and (1.4) are usually called the local log-Hölder condition and the decay log-Hölder condition, respectively. It is well known that for $1<p^{-} \leq p^{+}<$ $\infty$, both conditions are sufficient for the Hardy-Littlewood maximal operator to be bounded on variable Lebesgue spaces (see [14]). Moreover, in [45], Pick and Růžička gave an example of a space $L^{p(\cdot)}$ with $p \notin \mathcal{P}^{\log }\left(\mathbf{R}^{n}\right)$ where $M$ is not bounded.

Given two functions $f$ and $g$, by $\lesssim$ and $\gtrsim$ we will mean that there exists a positive constant $c$ such that $f \leq c g$ and $c f \geq g$, respectively. When both inequalities hold, that is, $f \lesssim g \lesssim f$, we will write it as $f \approx g$. 
As we said in the introduction, we are interested in maximal operators associated to Young functions. We will say that $\eta:[0, \infty) \rightarrow[0, \infty)$ is a Young function if it is increasing, convex, $\eta(0)=0$ and $\eta(t) \rightarrow \infty$ when $t \rightarrow \infty$. For simplicity, we will consider normalized Young functions, that is, $\eta(1)=1$. We also deal with submultiplicative Young functions, which means that $\eta(s t) \leq \eta(s) \eta(t)$ for every $s, t>$ 0 . If $\eta$ is a submultiplicative Young function, it follows that $\eta^{\prime}(t) \approx \eta(t) / t$.

Given a Young function $\eta$, we define the Orlicz space $L^{\eta}\left(\mathbf{R}^{n}\right)$ as the set of all measurable functions for which there exists a positive number $\lambda$ such that

$$
\int_{\mathbf{R}^{n}} \eta\left(\frac{|f(x)|}{\lambda}\right) d x \leq 1 .
$$

This definition induces the Luxemburg norm for this space, defined by

$$
\|f\|_{\eta}=\inf \left\{\lambda>0: \int_{\mathbf{R}^{n}} \eta\left(\frac{|f(x)|}{\lambda}\right) d x \leq 1\right\},
$$

and $\left(L^{\eta}\left(\mathbf{R}^{n}\right),\|\cdot\|_{\eta}\right)$ is a Banach space (see for instance [46]). Related with this norm, the Luxemburg average of a function $f$ over a ball $B$ is defined by

$$
\|f\|_{\eta, B}=\inf \left\{\lambda>0: \frac{1}{|B|} \int_{B} \eta\left(\frac{|f(y)|}{\lambda}\right) d y \leq 1\right\} .
$$

We will also need the following facts. Each Young function $\eta$ has an associated complementary Young function $\widetilde{\eta}$ satisfying $t \leq \eta^{-1}(t) \widetilde{\eta}^{-1}(t) \leq 2 t$ for every $t>0$. The following generalization of Hölder's inequality

$$
\int_{\mathbf{R}^{n}}|f g| \leq\|f\|_{\eta}\|g\|_{\widetilde{\eta}}
$$

holds and it is easy to check that we also have

$$
\frac{1}{|B|} \int_{B}|f g| \leq\|f\|_{\eta, B}\|g\|_{\tilde{\eta}, B}
$$

Moreover, there is a further generalization of the inequality above. If $\eta, \phi$ and $\psi$ are Young functions satisfying $\psi^{-1}(t) \phi^{-1}(t) \lesssim \eta^{-1}(t)$, then

$$
\|f g\|_{\eta, B} \lesssim\|f\|_{\phi, B}\|g\|_{\psi, B} .
$$

Associated to the average in (1.5), we define the generalized maximal operator $M_{\eta}$ by

$$
M_{\eta} f(x)=\sup _{B \ni x}\|f\|_{\eta, B}, \quad x \in \mathbf{R}^{n} .
$$

When $0 \leq \alpha<n$, the fractional version of the operator above is given by

$$
M_{\alpha, \eta} f(x)=\sup _{B \ni x}|B|^{\alpha / n}\|f\|_{\eta, B}, \quad x \in \mathbf{R}^{n} .
$$

Notice that when $\eta(t)=t, M_{\eta}$ and $M_{\alpha, \eta}$ are the Hardy-Littlewood and the fractional maximal operators, respectively.

In the following definition we introduce the class of weights related with the boundedness of the fractional maximal operator in variable Lebesgue spaces. By a weight we understand a non-negative and locally integrable function. 
1.6. Definition. Let $0 \leq \alpha<n$ and $p, q \in \mathcal{P}^{*}\left(\mathbf{R}^{n}\right)$ such that $1 / q(x)=1 / p(x)-$ $\alpha / n, p^{+}<n / \alpha$. We say that a weight $w \in A_{p(\cdot), q(\cdot)}$ if there exists a positive constant $C$ such that for every ball $B$, the inequality

$$
\left\|w \chi_{B}\right\|_{q(\cdot)}\left\|w^{-1} \chi_{B}\right\|_{p^{\prime}(\cdot)} \leq C|B|^{1-\frac{\alpha}{n}}
$$

holds.

When $\alpha=0$, we obtain the $A_{p(\cdot)}$ class given by Cruz-Uribe, Diening and Hästö in [9] that characterizes the boundedness of the Hardy-Littlewood maximal operator on $L_{w}^{p(\cdot)}\left(\mathbf{R}^{n}\right)$, that is, the measurable functions $f$ such that $f w \in L^{p(\cdot)}\left(\mathbf{R}^{n}\right)$.

\section{Main results}

In this section we state boundedness results for the maximal operators defined in section $\S 1$ on weighted variable Lebesgue spaces. As far as we know, our results are new even when $p$ is a constant exponent. We first consider $\eta$ to be a $L \log L$ type function.

2.1. Theorem. Let $w$ be a weight, $p \in \mathcal{P}^{\log }\left(\mathbf{R}^{n}\right)$ with $p^{-}>1$, and let $\eta$ be the Young function defined by $\eta(t)=t^{\beta}\left(1+\log ^{+} t\right)^{\gamma}$ with $1 \leq \beta<p^{-}$and $\gamma \geq 0$. Then, $M_{\eta}$ is bounded on $L_{w}^{p(\cdot)}\left(\mathbf{R}^{n}\right)$ if and only if $w^{\beta} \in A_{\frac{p(\cdot)}{\beta}}$.

Observe that when $\beta=1$ and $\gamma=k \in \mathbf{N}$, this is the expected result since it is well known that $M_{\eta}$ is equivalent to $M^{k+1}=M \circ \cdots \circ M$ iterated $k+1$ times (see [42]).

The following result gives the class of weights that characterizes the boundedness of the fractional maximal operator $M_{\alpha}$ on weighted variable Lebesgue spaces and extends the corresponding results given in [36] in the classical context. This result allows us to prove the corresponding result for the generalized maximal operator $M_{\alpha, \eta}$ (Theorem 2.3).

2.2. Theorem. Let $0 \leq \alpha<n$, w a weight and $p \in \mathcal{P}^{\log }\left(\mathbf{R}^{n}\right)$ with $1<p^{-} \leq$ $p^{+}<n / \alpha$. Let $q$ be the function defined by $1 / q(x)=1 / p(x)-\alpha / n$. Then, $M_{\alpha}$ is bounded from $L_{w}^{p(\cdot)}\left(\mathbf{R}^{n}\right)$ into $L_{w}^{q(\cdot)}\left(\mathbf{R}^{n}\right)$ if and only if $w \in A_{p(\cdot), q(\cdot)}$.

Thus, the fractional version of Theorem 2.1 is given in the next result.

2.3. Theorem. Let $0 \leq \alpha<n, w$ a weight, $p \in \mathcal{P}^{\log }\left(\mathbf{R}^{n}\right)$ such that $1<p^{-} \leq$ $p^{+}<n / \alpha$ and $1 / q(x)=1 / p(x)-\alpha / n$. Let $\eta(t)=t^{\beta}\left(1+\log ^{+} t\right)^{\gamma}$ with $1 \leq \beta<p^{-}$ and $\gamma \geq 0$. Then, $M_{\alpha, \eta}$ is bounded from $L_{w}^{p(\cdot)}\left(\mathbf{R}^{n}\right)$ into $L_{w}^{q(\cdot)}\left(\mathbf{R}^{n}\right)$ if and only if $w^{\beta} \in A_{\frac{p(\cdot)}{\beta}, \frac{q(\cdot)}{\beta}}$.

The next theorems involve a wider class of maximal operators associated to Young functions which satisfy certain Dini type condition, given in the following definition.

2.4. Definition. Let $1<q<\infty$. We say that a Young function $\eta$ satisfies the $B_{q}$ condition if there exists a positive constant $c$ such that

$$
\int_{c}^{\infty} \frac{\eta(t)}{t^{q}} \frac{d t}{t}<\infty .
$$


2.5. Theorem. Let $w$ be a weight, $p \in \mathcal{P}^{\log }\left(\mathbf{R}^{n}\right)$ and $1 \leq \beta<p^{-}$. Let $\eta$ be a Young function such that $\eta \in B_{\rho}$ for every $\rho>\beta$. If $w^{\beta} \in A_{\frac{p(\cdot)}{\beta}}$, then $M_{\eta}$ is bounded on $L_{w}^{p(\cdot)}\left(\mathbf{R}^{n}\right)$.

2.6. Theorem. Let $0 \leq \alpha<n$, w a weight, $p \in \mathcal{P}^{\log }\left(\mathbf{R}^{n}\right)$ with $1<p^{-} \leq p^{+}<$ $n / \alpha, 1 / q(x)=1 / p(x)-\alpha / n$ and $1 \leq \beta<p^{-}$. Let $\eta$ be a Young function such that $\eta^{1+\frac{\rho \alpha}{n-\alpha}} \in B_{\frac{\rho n}{n-\alpha}}$ for every $\rho>\beta(n-\alpha) /(n-\alpha \beta)$, and let $\phi$ be a Young function such that $\phi^{-1}(t) t^{\frac{\alpha}{n}} \lesssim \eta^{-1}(t)$ for every $t>0$. If $w^{\beta} \in A_{\frac{p(\cdot)}{\beta}, \frac{q(\cdot)}{\beta}}$, then $M_{\alpha, \eta}$ is bounded from $L_{w}^{p(\cdot)}\left(\mathbf{R}^{n}\right)$ into $L_{w}^{q(\cdot)}\left(\mathbf{R}^{n}\right)$.

2.7. Remark. Let us observe that Theorems 2.5 and 2.6 include the sufficiency results of Theorems 2.1 and 2.3, respectively. In fact, if $0 \leq \alpha<n$, then $\beta(1+$ $\rho \alpha /(n-\alpha))<\rho n /(n-\alpha)$ for every $\rho>\beta(n-\alpha) /(n-\alpha \beta)$ and thus, $\eta^{1+\frac{\rho \alpha}{n-\alpha}} \in B_{\frac{\rho n}{n-\alpha}}$, where $\eta(t)=t^{\beta}\left(1+\log ^{+} t\right)^{\gamma}$ with $1 \leq \beta<p^{-}$and $\gamma \geq 0$.

As we said in the introduction, the maximal operators $M_{\eta}$ are not of strong type $(1,1)$ for a large class of Young functions $\eta$. Moreover, they are not of weak type $(1,1)$. Nevertheless, for certain class of functions we can obtain local integrability properties in the spirit of Wiener's inequality. The remainder of this section is devoted to this type of results in the context of Musielak-Orlicz spaces.

Given $p \in \mathcal{P}^{*}\left(\mathbf{R}^{n}\right)$, a non-negative and bounded function $q$, and a Young function $\eta$, we denote by $\eta\left(L^{p(\cdot)}\right)(\log L)^{q(\cdot)}\left(\mathbf{R}^{n}\right)$ the Musielak-Orlicz space corresponding to the function $\Phi(t, x)=\eta\left(t^{p(x)}\right) \log (e+t)^{q(x)}$, and we write

$$
\|f\|_{\eta\left(L^{p(\cdot)}\right)(\log L)^{q(\cdot)}\left(\mathbf{R}^{n}\right)}=\|f\|_{\Phi(L, \cdot)\left(\mathbf{R}^{n}\right)}=\inf \left\{\lambda>0: \int_{\mathbf{R}^{n}} \Phi\left(x, \frac{|f(x)|}{\lambda}\right) d x \leq 1\right\} .
$$

For a given Musielak-Orlicz function $\Phi$ and a non-negative function $v$ we denote

$$
\|f\|_{\Phi(L, \cdot)\left(\mathbf{R}^{n}\right), v}=\inf \left\{\lambda>0: \int_{\mathbf{R}^{n}} \Phi\left(x, \frac{|f(x)|}{\lambda}\right) v(x) d x \leq 1\right\} .
$$

The next theorem is a generalization of Wiener's result proved in [48], for the case of the maximal operator $M_{\eta}$.

2.8. Theorem. Let $p \in \mathcal{P}^{*}\left(\mathbf{R}^{n}\right), w$ a weight and $\eta$ a Young function. Then, for every $\epsilon>0$, there exists a positive constant $C=C(\epsilon, p)$ such that for each ball $B$

$$
\int_{B} M_{\eta} f(x) w(x) d x \leq w(B)+C \int_{\mathbf{R}^{n}} \eta\left(|f(x)|^{p(x)}\right) \log (e+|f(x)|)^{q(x)} M w(x) d x,
$$

where $q(x)=\max \left\{\epsilon^{-1}(\epsilon+1-p(x)), 0\right\}$.

Particularly, if we consider certain class of Young functions $\eta$, we improved the result above in the following way.

2.9. Theorem. Let $p \in \mathcal{P}^{*}\left(\mathbf{R}^{n}\right), w$ a weight and $\eta$ a Young function such that $\eta \in B_{q}$ for each $q>1$. Then, for every $\epsilon>0$, there exists a positive constant $C=C(\epsilon, p)$ such that for each ball $B$

$\int_{B} M_{\eta} f(x) w(x) d x \leq w(B)+C \int_{\mathbf{R}^{n}}|f(x)|^{p(x)}\left[\eta^{\prime}(|f(x)|) \log (e+|f(x)|)\right]^{q(x)} M w(x) d x$,

where $q(x)=\max \left\{\epsilon^{-1}(\epsilon+1-p(x)), 0\right\}$. 
2.10. Corollary. Let $p \in \mathcal{P}^{*}\left(\mathbf{R}^{n}\right), w$ a weight and $\eta$ a Young function. Then, for every $\epsilon>0$, there exists a positive constant $C=C(\epsilon, p)$ such that for each ball $B$ with $w(B) \leq 1$

$$
\left\|M_{\eta} f\right\|_{L^{1}(B), w} \leq C\|f\|_{\eta\left(L^{p(\cdot)}\right)(\log L)^{q(\cdot)}\left(\mathbf{R}^{n}\right), M w}
$$

where $q(x)=\max \left\{\epsilon^{-1}(\epsilon+1-p(x)), 0\right\}$.

By requiring additional properties on the exponent $p$, we obtained more integrability for the maximal operator $M_{\eta}$.

2.11. Theorem. Let $p \in \mathcal{P}^{\log }\left(\mathbf{R}^{n}\right)$ and $w$ a weight. Let $\eta$ be a submultiplicative Young function such that $\eta \in B_{p^{-}}$if $p^{-}>1$, or $\eta \in B_{q}$ for every $1<q<\infty$ if $p^{-}=1$. Then, given $0<\epsilon<1$, there exist functions $r, q \in \mathcal{P}^{\log }\left(\mathbf{R}^{n}\right)$ such that for every ball $B$ and every function $f$ with $\int_{\{|f|>1\}} \eta\left(|f(x)|^{p(x)}\right) d x \leq 1$

$$
\int_{B} M_{\eta} f(x)^{r(x)} w(x) d x \leq w(B)+C \int_{\mathbf{R}^{n}} \eta\left(|f(x)|^{p(x)}\right) \log (e+|f(x)|)^{q(x)} M w(x) d x
$$

and such that the following properties hold,

(i) $r(x)=p(x)$ whenever $p(\cdot)$ takes values outside the range $(1,1+\epsilon)$, and $1<r(x)<p(x)$ if $p(\cdot)$ takes values on $(1,1+\epsilon)$,

(ii) $0 \leq q(x) \leq 1, q(x)=1$ if $p(x)=1$ and $q(x)=0$ if $p(x) \geq 1+\epsilon$.

As a consequence we obtain the following result.

2.12. Corollary. Let $p$ and $\eta$ be as in the hypothesis of Theorem 2.11. Given a ball $B$, let $w$ be a weight such that $w(B) \leq 1$. Then, given $0<\epsilon<1$, there exist functions $r, q \in \mathcal{P}^{\log }\left(\mathbf{R}^{n}\right)$ having the properties (i) and (ii) of Theorem 2.11 such that

$$
\left\|M_{\eta} f\right\|_{L^{r(\cdot)}(B), w} \leq C\|f\|_{\eta\left(L^{p(\cdot)}\right)(\log L)^{q(\cdot)}\left(\mathbf{R}^{n}\right), M w} .
$$

2.13. Remark. Note that by requiring $\eta \in B_{q}$ for every $q>1$ in Theorem 2.11 and Corollary 2.12, we can obtain better results by applying Theorem 2.9:

$$
\begin{aligned}
& \int_{B} M_{\eta} f(x)^{r(x)} w(x) d x \\
& \leq w(B)+C \int_{B}|f(x)|^{p(x)}\left[\eta^{\prime}(|f(x)|) \log (e+|f(x)|)\right]^{q(x)} M w(x) d x
\end{aligned}
$$

and

$$
\left\|M_{\eta} f\right\|_{L^{r(\cdot)}(B), w} \leq C\|f\|_{L^{p(\cdot)}\left(\eta^{\prime}(L) \log L\right)^{q(\cdot)}(B), M w} .
$$

For example, if $\eta(t)=t\left(1+\log ^{+} t\right)^{k}, k \in \mathbf{N} \cup\{0\}$, it is known that $M_{\eta} \approx M^{k+1}$. By considering $p^{-}>1$ and $w=1$, Theorem 2.9 contains the boundedness of $M^{k+1}$ in $L^{p(\cdot)}(B)$ as a particular case.

2.14. Remark. The unweighted case for the Hardy-Littlewood maximal operator of Theorems 2.8 and 2.11 and Corollaries 2.10 and 2.12 were proved in [11]. These results are particular cases of our results when $\eta(t)=t$ and $w=1$.

The following estimates are the fractional versions of Theorem 2.8 and Corollary 2.10. Since the fractional maximal operator $M_{\alpha}$ in not of strong type $(1, n /(n-$ $\alpha)$ ) and from the fact that $M_{\alpha, \eta} \gtrsim M_{\alpha}$ it follows that the operator $M_{\alpha, \eta}$ is not of strong type $(1, n /(n-\alpha))$ either. In view of this, we were interested in studying the 
local integrability in $L^{\frac{n}{n-\alpha}}$ for the generalized fractional maximal operator $M_{\alpha, \eta}$ with weights.

2.15. Theorem. Let $0 \leq \alpha<n$ and let $w$ be a weight. Let $p \in \mathcal{P}^{*}\left(\mathbf{R}^{n}\right)$, let $\eta$ be a Young function and let $\phi$ be another Young function satisfying $\phi^{-1}(t) t^{\alpha / n} \lesssim \eta^{-1}(t)$. Then, for every $\epsilon>0$, there exists a positive constant $C=C(\epsilon, p)$ such that for every ball $B$

$$
\begin{aligned}
& \left(\int_{B} M_{\alpha, \eta} f(x)^{\frac{n}{n-\alpha}} w(x) d x\right)^{1-\alpha / n} \\
& \leq C\left(w(B)+\int_{\mathbf{R}^{n}} \psi\left(|f(x)|^{p(x)}\right) \log (e+|f(x)|)^{r(x)} M w(x) d x\right),
\end{aligned}
$$

whenever $f \in L^{p(\cdot)}\left(\mathbf{R}^{n}\right)$ with $\|f\|_{p(\cdot)} \leq 1$. Here $r(x)=\max \left\{\epsilon^{-1}(\epsilon+1-s(x)), 0\right\}$ and $\psi(t)=\phi\left(t^{1-\alpha / n}\right)$.

2.16. Corollary. Let $0 \leq \alpha<n$. Let $p, \eta$ and $\phi$ as in Theorem 2.15. Then, for every $\epsilon>0$, there exists a positive constant $C=C(\epsilon, p)$ such that for every ball $B$ with $w(B) \leq 1$

$$
\left\|M_{\alpha, \eta} f\right\|_{L^{\frac{n}{n-\alpha}}(B), w} \leq C\|f\|_{\psi\left(L^{p(\cdot)}\right)(\log L)^{r(\cdot)}\left(\mathbf{R}^{n}\right), M w}
$$

whenever $f \in L^{p(\cdot)}\left(\mathbf{R}^{n}\right)$ with $\|f\|_{p(\cdot)} \leq 1$. Here $r(x)=\max \left\{\epsilon^{-1}(\epsilon+1-s(x)), 0\right\}$ and $\psi(t)=\phi\left(t^{1-\alpha / n}\right)$.

\section{Applications of weighted strong type inequalities}

3.1. Singular integral operators. As we said before, the generalized maximal operators $M_{\eta}$ control a large class of operators such as singular integrals and their commutators. In several cases this control is given by an inequality of the type

$$
M_{\delta}^{\sharp}(T f)(x) \leq C_{\delta} M_{\eta} f(x),
$$

where

$$
M^{\sharp} f(x)=\sup _{B \ni x} \inf _{a \in \mathbf{R}} f_{B}|f(y)-a| d y \approx \sup _{B \ni x} f_{B}\left|f(y)-m_{B} f\right| d y
$$

and, for $\delta>0, M_{\delta}^{\sharp} f=M^{\sharp}\left(|f|^{\delta}\right)^{\frac{1}{\delta}}$.

Let $T$ be a singular integral operator of convolution type with kernel $K$, that is, $T$ is bounded on $L^{2}\left(\mathbf{R}^{n}\right)$ and

$$
T f(x)=\mathrm{p} \cdot \mathrm{v} \cdot \int_{\mathbf{R}^{n}} K(x-y) f(y) d y,
$$

where $K$ is a measurable function defined away from 0 . In order to describe the behaviour of $T$ in the variable Lebesgue spaces we assume some smoothness condition on $K$. We first consider the so-called Hörmander condition: we say that $K$ satisfies the $L^{1}$-Hörmander condition if there exist $c>1$ and $C_{1}>0$ such that

$$
\int_{|x|>c|y|}|K(x-y)-K(x)| d x \leq C_{1}, \quad y \in \mathbf{R}^{n} .
$$

This is the weakest condition we will be dealing with and we refer to it as $H_{1}$ condition. The classical Lipschitz condition is denoted by $H_{\infty}^{*}$. We say that $K \in H_{\infty}^{*}$ if 
there exist $\theta, C>0$ and $c>1$ such that

$$
|K(x-y)-K(x)| \leq C \frac{|y|^{\theta}}{|x|^{\theta+n}}, \quad|x|>c|y| .
$$

It is easy to note that $H_{\infty}^{*} \subset H_{1}$. Among these classes, we can consider the $L^{r}$ Hörmander conditions, $1 \leq r \leq \infty$ or, more generally, the $L^{\mathcal{A}}$ - Hörmander condition associated to a Young function $\mathcal{A}$. Given a Young function $\mathcal{A}$ we write

$$
\|f\|_{\mathcal{A},|x| \sim s}=\left\|f \chi_{\{|x| \sim s\}}\right\|_{\mathcal{A}, B(0,2 s)}
$$

where $|x| \sim s$ means $s<|x| \leq 2 s$. We say that the kernel $K$ satisfies the $L^{\mathcal{A}_{-}}$ Hörmander condition or simply $K \in H_{\mathcal{A}}$ if there exist $c \geq 1$ and $C_{\mathcal{A}}>0$ such that for any $y \in \mathbf{R}^{n}$ and $R>c|y|$,

$$
\sum_{m=1}^{\infty}\left(2^{m} R\right)^{n}\|K(\cdot-y)-K(\cdot)\|_{\mathcal{A},|x| \sim 2^{m} R} \leq C_{\mathcal{A}} .
$$

When $\mathcal{A}(t)=t^{r}$, we obtain the $L^{r}$-Hörmander conditions and we denote $K \in H_{r}$. All the conditions above satisfy the following relations

$$
H_{\infty}^{*} \subset H_{\infty} \subset H_{\mathcal{A}} \subset H_{1}
$$

for any Young function $\mathcal{A}$. Particularly, if $1<r<s<\infty$, then $H_{s} \subset H_{r}$ (for more information about this conditions, see [34]).

Related with the pointwise estimate (3.1), in [35] the authors proved the following result.

3.2. Theorem. Let $T$ be a singular integral operator with kernel $K \in H_{\mathcal{A}}$. Then for any $0<\delta<1$, there exists a positive constant $C_{\delta}$ such that

$$
M_{\delta}^{\sharp}(T f)(x) \leq C_{\delta} M_{\widetilde{\mathcal{A}}} f(x),
$$

where $\widetilde{\mathcal{A}}$ is the complementary Young function associated to $\mathcal{A}$.

As a consequence of the theorem above and Theorem 2.5 we get the following result related to the boundedness of the operator $T$ in $L_{w}^{p(\cdot)}\left(\mathbf{R}^{n}\right)$. By $L_{c}^{\infty}\left(\mathbf{R}^{n}\right)$ we will denote the set of bounded functions with compact support.

3.3. Theorem. Let $p \in \mathcal{P}^{\log }\left(\mathbf{R}^{n}\right), 1 \leq \beta<p^{-}$and let $\mathcal{A}$ be a Young function such that $\widetilde{\mathcal{A}} \in B_{\rho}$, for every $\rho>\beta$. Let $T$ be a singular integral operator with kernel $K \in H_{\mathcal{A}}$. If $w^{\beta} \in A_{\frac{p(\cdot)}{\beta}}$ then

$$
\|w T f\|_{p(\cdot)} \lesssim\|w f\|_{p(\cdot)}
$$

for every $f \in L_{c}^{\infty}\left(\mathbf{R}^{n}\right)$, whenever the left-hand side is finite.

In order to prove the result above, we will be using the following lemmas proved in [29] and [26], respectively.

3.4. Lemma. If $h \in L^{r(\cdot)}\left(\mathbf{R}^{n}\right)$ and $r^{+}<\infty$, the following inequalities hold

$$
\frac{1}{2}\|h\|_{r(\cdot)} \leq \sup _{\|g\|_{r^{\prime}(\cdot)} \leq 1} \int_{\mathbf{R}^{n}}|h g| d x \leq 2\|h\|_{r(\cdot)} .
$$


3.5. Lemma. There exists a positive constant $C$ such that for every weight $w$ and every non-negative measurable function $f$ such that $|\{x: f(x)>\lambda\}|<\infty$ for every $\lambda>0$, the following inequality holds

$$
\int_{\mathbf{R}^{n}} f w d x \leq C \int_{\mathbf{R}^{n}} M^{\sharp} f M w d x .
$$

Proof of Theorem 3.3. Let $0<\delta<1$ and $f \in L_{c}^{\infty}\left(\mathbf{R}^{n}\right)$. Let us observe that $w^{\beta} \in$ $A_{\frac{p(\cdot)}{\beta}}$ implies $f \in L_{w}^{p(\cdot)}\left(\mathbf{R}^{n}\right)$ because $w \in L_{\text {loc }}^{p(\cdot)}\left(\mathbf{R}^{n}\right)$, that is, $w^{p(\cdot)}$ is a locally integrable function. Now, if $r(\cdot)=p(\cdot) / \delta$, by Lemma 3.4 and the fact that $|T f|^{\delta} \in L_{w^{\delta}}^{r(\cdot)}\left(\mathbf{R}^{n}\right)$, we have

$$
\|w T f\|_{p(\cdot)}=\left\|w^{\delta}|T f|^{\delta}\right\|_{r(\cdot)}^{1 / \delta} \lesssim \sup _{\left\|w^{-\delta} g\right\|_{r^{\prime}(\cdot)} \leq 1}\left(\int_{\mathbf{R}^{n}}|T f|^{\delta}|g| d x\right)^{1 / \delta}
$$

By the hypothesis on $T$ we have that $\left|\left\{x \in \mathbf{R}^{n}:|T f(x)|>\lambda\right\}\right|<\infty$ for every $\lambda>0$. Then by applying Lemma (3.5) with $f$ and $w$ replaced by $|T f|^{\delta} \geq 0$ and $|g|$ respectively, and the generalized Hölder inequality we obtain that

$$
\begin{aligned}
\|w T f\|_{p(\cdot)} & \lesssim \sup _{\left\|w^{-\delta} g\right\|_{r^{\prime}(\cdot)} \leq 1}\left(\int_{\mathbf{R}^{n}} M^{\sharp}\left(|T f|^{\delta}\right) M g d x\right)^{1 / \delta} \\
& \lesssim \sup _{\left\|w^{-\delta} g\right\|_{r^{\prime}(\cdot)} \leq 1}\left\|w^{\delta} M^{\sharp}\left(|T f|^{\delta}\right)\right\|_{r(\cdot)}^{1 / \delta}\left\|w^{-\delta} M g\right\|_{r^{\prime}(\cdot)}^{1 / \delta} \\
& =\sup _{\left\|w^{-\delta} g\right\|_{r^{\prime}(\cdot)} \leq 1}\left\|w M_{\delta}^{\sharp}(|T f|)\right\|_{p(\cdot)}\left\|w^{-\delta} M g\right\|_{r^{\prime}(\cdot)}^{1 / \delta} .
\end{aligned}
$$

Since $w^{\beta} \in A_{\frac{p(\cdot)}{\beta}}$ for some $1 \leq \beta<p^{-}$implies that $w^{\delta} \in A_{r(\cdot)}$ (see (4.4)), which is equivalent to $w^{-\delta} \in A_{r^{\prime}(\cdot)}$, by Theorems 2.5 and 3.2 we obtain that

$$
\|w T f\|_{p(\cdot)} \lesssim \sup _{\left\|w^{-\delta} g\right\|_{r^{\prime}(\cdot)} \leq 1}\left\|w M_{\delta}^{\sharp}(|T f|)\right\|_{p(\cdot)}\left\|w^{-\delta} g\right\|_{r^{\prime}(\cdot)}^{1 / \delta} \lesssim\left\|w M_{\widetilde{\mathcal{A}}} f\right\|_{p(\cdot)} \lesssim\|w f\|_{p(\cdot)}
$$

3.6. Remark. Notice that if $K \in H_{\infty}^{*}$ then we can take $w \in A_{p(\cdot)}$ in Theorem 3.3 since it is well known that (3.1) holds with $M_{\eta}=M$. When $K \in H_{r}$, for some $\left(p^{-}\right)^{\prime}<r \leq \infty$ then $\tilde{\mathcal{A}}=t^{r^{\prime}}$ and we can take $w^{r^{\prime}} \in A_{\frac{p(\cdot)}{r^{\prime}}}$ in the same theorem in order to get the boundedness result for the operator $T$. In [34] the authors present several examples of singular integral operators with kernels satisfying the $L^{\mathcal{A}}$-Hörmander conditions described above for certain Young functions $\mathcal{A}$, including, for example, homogeneous singular integrals and Fourier multipliers.

The higher order commutators with $B M O$ symbols of the singular integral operators of the type described above were also considered in [34]. Given $T$ and $b \in B M O$ we define the $k$-th order commutator, $k \in \mathbf{N} \cup\{0\}$, by

$$
T_{b}^{k} f(x)=\int_{\mathbf{R}^{n}}(b(x)-b(y))^{k} K(x-y) f(y) d y .
$$

In order to study these operators the authors define the corresponding smoothness condition depending on a Young function $\mathcal{A}$ and the order of the commutators.

Let $\mathcal{A}$ be a Young function and $k \in \mathbf{N}$. We say that the kernel $K$ satisfies the $L^{\mathcal{A}, k}$-Hörmander condition or that $K \in H_{\mathcal{A}, k}$ if there exist $c \geq 1$ and $C>0$, 
depending on $\mathcal{A}$ and $k$ such that for every $y \in \mathbf{R}^{n}$ and $R>c|y|$

$$
\sum_{m=1}^{\infty}\left(2^{m} R\right)^{n} m^{k}\|K(\cdot-y)-K(\cdot)\|_{\mathcal{A},|x| \sim 2^{m} R} \leq C .
$$

In [34] the authors also proved the following result.

3.7. Theorem. Let $\mathcal{A}$ and $\mathcal{B}$ be Young functions such that $\widetilde{\mathcal{A}}^{-1}(t) \mathcal{B}^{-1}(t)(\log t)^{k}$ $\leq t$ for $t \geq t_{0}>1$. If $T$ is a singular integral operator with kernel $K \in H_{\mathcal{B}} \cap H_{\mathcal{A}, k}$, for any $b \in B M O, 0<\delta<\epsilon<1$ and $k \in \mathbf{N}$, there exists $C=C_{\delta, \epsilon}$ such that

$$
M_{\delta}^{\sharp}\left(T_{b}^{k} f\right)(x) \leq C \sum_{j=0}^{k-1}\|b\|_{B M O}^{k-j} M_{\epsilon}\left(T_{b}^{j} f\right)(x)+C\|b\|_{B M O}^{k} M_{\widetilde{\mathcal{A}}} f(x)
$$

Then, we have the following result for $T_{b}^{k}$.

3.8. Theorem. Let $p \in \mathcal{P}^{\log }\left(\mathbf{R}^{n}\right), 1 \leq \beta<p^{-}$. Let $\mathcal{A}$ and $\mathcal{B}$ be Young functions such that $\widetilde{\mathcal{A}}^{-1}(t) \mathcal{B}^{-1}(t)(\log t)^{k} \leq t$ for $t \geq t_{0}>1, k \in \mathbf{N}$ and $\widetilde{\mathcal{A}} \in B_{\rho}$, for every $\rho>\beta$. Let $b \in B M O$ and $T_{b}^{k}$ be the $k$-th order commutator of the singular integral operator $T$ with kernel $K \in H_{\mathcal{B}} \cap H_{\mathcal{A}, k}$. If $w^{\beta} \in A_{\frac{p(\cdot)}{\beta}}$, then

$$
\left\|w T_{b}^{k} f\right\|_{p(\cdot)} \lesssim\|b\|_{B M O}^{k}\|w f\|_{p(\cdot)}
$$

for every $f \in L_{c}^{\infty}\left(\mathbf{R}^{n}\right)$, whenever the left-hand side is finite.

3.9. Remark. When $k=0$, Theorems 3.7 and 3.8 are Theorems 3.2 and 3.3.

Proof. We use an induction argument. The result holds for the case $k=0$ which is nothing but Theorem 3.3, and suppose it is true for every $0 \leq j \leq k-1$. Without loss of generality we can consider $\|b\|_{B M O}=1$. Let $f \in L_{c}^{\infty}\left(\mathbf{R}^{n}\right), 0<\delta<1$ and $r(\cdot)=p(\cdot) / \delta$. Thus, proceeding as in the proof of Theorem 3.3 and applying Theorem 3.7 we obtain

$$
\begin{aligned}
\left\|w T_{b}^{k} f\right\|_{p(\cdot)} & \lesssim \sup _{\left\|w^{-\delta} g\right\|_{r^{\prime}(\cdot)} \leq 1}\left(\int_{\mathbf{R}^{n}} M^{\sharp}\left(\left|T_{b}^{k} f\right|^{\delta}\right) M g d x\right)^{1 / \delta} \\
& =\sup _{\left\|w^{-\delta} g\right\|_{r^{\prime}(\cdot)} \leq 1}\left\|w M_{\delta}^{\sharp}\left(\left|T_{b}^{k} f\right|\right)\right\|_{p(\cdot)}\left\|w^{-\delta} g\right\|_{r^{\prime}(\cdot)}^{1 / \delta} \\
& \lesssim \sum_{j=0}^{k-1}\left\|w M_{\epsilon}\left(T_{b}^{j} f\right)\right\|_{p(\cdot)}+\left\|w M_{\widetilde{\mathcal{A}}} f\right\|_{p(\cdot)}
\end{aligned}
$$

From the hypothesis on $w$ we have that $w^{\epsilon} \in A_{\frac{p(\cdot)}{\epsilon}}$ for $0<\epsilon<1$ and thus, by applying the boundedness result for the Hardy-Littlewood maximal operator proved in [9], the inductive hypothesis and Theorem 2.5 we obtain that

$$
\left\|w T_{b}^{k} f\right\|_{p(\cdot)} \lesssim \sum_{j=0}^{k-1}\left\|w T_{b}^{j} f\right\|_{p(\cdot)}+\left\|w M_{\widetilde{\mathcal{A}}} f\right\|_{p(\cdot)} \lesssim\|w f\|_{p(\cdot)} .
$$

3.2. Fractional integral operators. In [4] the authors consider fractional operators of the type

$$
T_{\alpha} f(x)=\int_{\mathbf{R}^{n}} K_{\alpha}(x-y) f(y) d y, \quad 0 \leq \alpha<n,
$$


where the kernel $K_{\alpha}$ satisfies a size type condition and a fractional Hörmander condition. The size condition is called $S_{\alpha}$ : we say that $K_{\alpha} \in S_{\alpha}$ if there exists $C>0$ such that

$$
\int_{|x| \sim s}\left|K_{\alpha}(x)\right| d x \leq C s^{\alpha}
$$

The smoothness condition is defined as follows. We say that $K_{\alpha} \in H_{\alpha, \mathcal{A}}$ if there exist $c \geq 1$ and $C>0$ such that for every $y \in \mathbf{R}^{n}$ and $R>c|y|$

$$
\sum_{m=1}^{\infty}\left(2^{m} R\right)^{n-\alpha}\left\|K_{\alpha}(\cdot-y)-K_{\alpha}(\cdot)\right\|_{\mathcal{A},|x| \sim 2^{m} R} \leq C .
$$

The following result was proved in [4].

3.10. Theorem. Let $T_{\alpha}$ be a fractional operator with kernel $K_{\alpha} \in S_{\alpha} \cap H_{\alpha, \mathcal{A}}$. Then, for any $0<\delta<1$, there exists a positive constant $C_{\delta}$ such that

$$
M_{\delta}^{\sharp}\left(T_{\alpha} f\right)(x) \leq C_{\delta} M_{\alpha, \widetilde{\mathcal{A}}} f(x) .
$$

Then, the corresponding boundedness result for $T_{\alpha}$ in weighted variable Lebesgue spaces is the following.

3.12. Theorem. Let $0 \leq \alpha<n, p \in \mathcal{P}^{\log }\left(\mathbf{R}^{n}\right)$ with $1<p^{-} \leq p^{+}<n / \alpha$, $1 / q(x)=1 / p(x)-\alpha / n$ and $1 \leq \beta<p^{-}$. Let $\mathcal{A}$ be a Young function satisfying $\widetilde{\mathcal{A}}^{1+\frac{\rho \alpha}{n-\alpha}} \in B_{\frac{\rho n}{n-\alpha}}$ for every $\rho>\beta(n-\alpha) /(n-\alpha \beta)$ and let $\phi$ be a Young function such that $\phi^{-1}(t) t^{\frac{\alpha}{n}} \lesssim \widetilde{\mathcal{A}}^{-1}(t)$ for every $t>0$. Let $T_{\alpha}$ be a fractional operator with kernel $K \in S_{\alpha} \cap H_{\alpha, \mathcal{A}}$. If $w^{\beta} \in A_{\frac{p(\cdot)}{\beta}, \frac{q(\cdot)}{\beta}}$, then

$$
\left\|w T_{\alpha} f\right\|_{q(\cdot)} \lesssim\|w f\|_{p(\cdot)}
$$

for every $f \in L_{c}^{\infty}\left(\mathbf{R}^{n}\right)$ such that $\left|\left\{x \in \mathbf{R}^{n}:\left|T_{\alpha} f(x)\right|>\lambda\right\}\right|<\infty$ for each $\lambda>0$ and whenever the left-hand side is finite.

Proof. Let $r(\cdot)=q(\cdot) / \delta, 0<\delta<1$. By the hypothesis over $T_{\alpha}$, we can proceed as before, by applying Lemma 3.5 and Theorem 3.10. Moreover, since $w^{\beta} \in A_{\frac{p(\cdot)}{\beta}, \frac{q(\cdot)}{\beta}}$ is equivalent to $w^{\gamma} \in A_{\frac{q(\cdot)}{\gamma}}$ for $\gamma=\frac{\beta n}{n-\alpha \beta}$, we have that $w^{\delta} \in A_{\frac{q(\cdot)}{\delta}}$ (see (4.2) and (4.4)). Thus, by the boundedness results obtained in [9] for $M$ we have

$$
\begin{aligned}
\left\|w T_{\alpha} f\right\|_{q(\cdot)} & \lesssim \sup _{\left\|w^{-\delta} g\right\|_{r^{\prime}(\cdot)} \leq 1}\left(\int_{\mathbf{R}^{n}} M^{\sharp}\left(\left|T_{\alpha} f\right|^{\delta}\right) M g d x\right)^{1 / \delta} \\
& \lesssim \sup _{\left\|w^{-\delta} g\right\|_{r^{\prime}(\cdot)} \leq 1}\left\|w^{\delta} M^{\sharp}\left(\left|T_{\alpha} f\right|^{\delta}\right)\right\|_{r(\cdot)}^{1 / \delta}\left\|w^{-\delta} M g\right\|_{r^{\prime}(\cdot)}^{1 / \delta} \\
& \lesssim\left\|w M_{\delta}^{\sharp}\left(\left|T_{\alpha} f\right|\right)\right\|_{q(\cdot)} \lesssim\left\|w M_{\alpha, \widetilde{\mathcal{A}}} f\right\|_{q(\cdot) .}
\end{aligned}
$$

Finally, by the properties on $\mathcal{A}$, Theorem 2.6 gives the desired result.

Given $b \in B M O$, the $k$-th order commutator of $T_{\alpha}$ for $k \in \mathbf{N} \cup\{0\}$ is defined by

$$
T_{\alpha, b}^{k} f(x)=\int_{\mathbf{R}^{n}}(b(x)-b(y))^{k} K_{\alpha}(x-y) f(y) d y, \quad 0 \leq \alpha<n .
$$

When $k=0, T_{\alpha, b}^{k}=T_{\alpha}$. We will now suppose that the kernel $K_{\alpha}$ satisfies the condition $H_{\alpha, \mathcal{A}, k}$, which means that there exist $c \geq 1$ and $C>0$ such that for every 
$y \in \mathbf{R}^{n}$ and $R>c|y|$

$$
\sum_{m=1}^{\infty}\left(2^{m} R\right)^{n-\alpha} m^{k}\left\|K_{\alpha}(\cdot-y)-K_{\alpha}(\cdot)\right\|_{\mathcal{A},|x| \sim 2^{m} R} \leq C .
$$

In [4] the authors also proved an estimate in the spirit of (3.11) for $T_{\alpha, b}^{k}$, which is given in the following result.

3.13. Theorem. Let $\mathcal{A}$ and $\mathcal{B}$ be Young functions such that $\widetilde{\mathcal{A}}^{-1}(t) \mathcal{B}^{-1}(t) \leq$ $t /\left(1+\log ^{+} t\right)^{k}$. If $T_{\alpha}$ is a fractional integral operator with kernel $K \in S_{\alpha} \cap H_{\alpha, \mathcal{B}} \cap$ $H_{\alpha, \mathcal{A}, k}$, for any $b \in B M O, 0<\delta<\epsilon<1$ and $k \in \mathbf{N}$, there exists $C=C_{\delta, \epsilon}$ such that

$$
M_{\delta}^{\sharp}\left(T_{\alpha, b}^{k} f\right)(x) \leq C \sum_{j=0}^{k-1}\|b\|_{B M O}^{k-j} M_{\epsilon}\left(T_{\alpha, b}^{j} f\right)(x)+C\|b\|_{B M O}^{k} M_{\alpha, \widetilde{\mathcal{A}}} f(x) .
$$

Then, with the same arguments used in this section, we obtain the next boundedness result for $T_{\alpha, b}^{k}$. We omit the details of the proof.

3.14. Theorem. Let $0 \leq \alpha<n, p \in \mathcal{P}^{\log }\left(\mathbf{R}^{n}\right)$ with $1<p^{-} \leq p^{+}<n / \alpha$, $1 / q(x)=1 / p(x)-\alpha / n$ and $1 \leq \beta<p^{-}$. Let $\mathcal{A}$ and $\mathcal{B}$ be Young functions such that $\widetilde{\mathcal{A}}^{-1}(t) \mathcal{B}^{-1}(t) \leq t /\left(1+\log ^{+} t\right)^{k}$ and $\widetilde{\mathcal{A}}^{1+\frac{\rho \alpha}{n-\alpha}} \in B_{\frac{\rho n}{n-\alpha}}$ for every $\rho>\beta(n-\alpha) /(n-\alpha \beta)$ and let $\phi$ be a Young function such that $\phi^{-1}(t) t^{\frac{\alpha}{n}} \lesssim \widetilde{\mathcal{A}}^{-1}(t)$ for every $t>0$. Let $b \in B M O$ and $T_{\alpha, b}^{k}$ be the $k$-th order commutator of the fractional integral operator $T_{\alpha}$ with kernel $K \in S_{\alpha} \cap H_{\alpha, \mathcal{B}} \cap H_{\alpha, \mathcal{A}, k}$. If $w^{\beta} \in A_{\frac{p(\cdot)}{\beta}, \frac{q(\cdot)}{\beta}}$, then

$$
\left\|w T_{\alpha, b}^{k} f\right\|_{q(\cdot)} \lesssim\|b\|_{B M O}^{k}\|w f\|_{p(\cdot)}
$$

for every $f \in L_{c}^{\infty}\left(\mathbf{R}^{n}\right)$ such that $\left|\left\{x \in \mathbf{R}^{n}:\left|T_{\alpha, b}^{k} f(x)\right|>\lambda\right\}\right|<\infty$ for each $\lambda>0$ and whenever the left-hand side is finite.

\section{Auxiliary results}

In this section we give the basic tools in order to get our main results. The next lemma gives some relations between the $A_{p(\cdot)}$ and the $A_{p(\cdot), q(\cdot)}$ classes.

4.1. Lemma. Let $0 \leq \alpha<n, 0<\delta<1$, and let $w$ be a weight. Let $p, q, s \in$ $\mathcal{P}^{*}\left(\mathbf{R}^{n}\right)$ such that $1 / q(x)=1 / p(x)-\alpha / n$ and $s(x)=(1-\alpha / n) q(x)$ with $1<p^{-} \leq$ $p^{+}<n / \alpha$.

(4.2) For every $1 \leq \beta \leq p^{-}, w^{\beta} \in A_{\frac{p(\cdot)}{\beta}, \frac{q(\cdot)}{\beta}}$ if and only if $w^{\frac{\beta n}{n-\alpha \beta}} \in A_{q(\cdot)\left(\frac{1}{\beta}-\frac{\alpha}{n}\right)}$.

Particularly, when $\beta=1, w \in A_{p(\cdot), q(\cdot)}$ if and only if $w^{\frac{n}{n-\alpha}} \in A_{s(\cdot)}$.

(4.3) If $w \in A_{p(\cdot), q(\cdot)}$, then $w^{\delta} \in A_{\frac{q(\cdot)}{\delta}}$.

(4.4) Let $1 \leq r \leq q^{-}$. If $w^{r} \in A_{\frac{q(\cdot)}{r}}$, then $w^{\delta} \in A_{\frac{q(\cdot)}{\delta}}$.

4.5. Remark. Noticing that $q(\cdot) / s(\cdot)=n /(n-\alpha)$, it follows that (4.2) is an extension of the results proved in [36].

Proof of Lemma 4.1. Let us prove (4.2). Notice that $0<\frac{n-\alpha \beta}{n} \leq q^{-}\left(\frac{n-\alpha \beta}{n \beta}\right)$ since $\beta \leq p^{-} \leq q^{-}$. Then

$$
\left\|w^{\frac{n \beta}{n-\alpha \beta}} \chi_{B}\right\|_{q(\cdot)\left(\frac{1}{\beta}-\frac{\alpha}{n}\right)}=\left\|w^{\beta} \chi_{B}\right\|_{q(\cdot)\left(\frac{n-\alpha \beta}{n \beta}\right)\left(\frac{n}{n-\alpha \beta}\right)}^{\frac{n}{n-\alpha \beta}}=\left\|w^{\beta} \chi_{B}\right\|_{\frac{q(\cdot)}{\beta}}^{\frac{n}{n-\alpha \beta}} .
$$


On the other hand, since $q(\cdot) n /(q(\cdot)(n-\alpha \beta)-n \beta)=(p(\cdot) / \beta)^{\prime}$ we obtain that

$$
\left\|w^{\frac{-n \beta}{n-\alpha \beta}} \chi_{B}\right\|_{\frac{q(\cdot)(n-\alpha \beta)}{q(\cdot)(n-\alpha \beta)-n \beta}}=\left\|w^{-\beta} \chi_{B}\right\|_{\frac{q(\cdot) n}{q(\cdot)(n-\alpha \beta)-n \beta}}^{\frac{n}{n-\alpha \beta}}=\left\|w^{-\beta} \chi_{B}\right\|_{\left(\frac{p(\cdot)}{\beta}\right)^{\prime}}^{\frac{n}{n-\alpha \beta}} .
$$

Let us first suppose that $w^{\beta} \in A_{\frac{p(\cdot)}{\beta}, \frac{q(\cdot)}{\beta}}$. Hence, from (4.6) and (4.7),

$$
\begin{aligned}
\left\|w^{\frac{n \beta}{n-\alpha \beta}} \chi_{B}\right\|_{q(\cdot)\left(\frac{1}{\beta}-\frac{\alpha}{n}\right)}\left\|w^{\frac{-n \beta}{n-\alpha \beta}} \chi_{B}\right\|_{\frac{q(\cdot)(n-\alpha \beta)}{q(\cdot)(n-\alpha \beta)-n \beta}} & =\left\|w^{\beta} \chi_{B}\right\|_{\frac{q(\cdot)}{\beta}}^{\frac{n}{n-\alpha \beta}}\left\|w^{-\beta} \chi_{B}\right\|_{\left(\frac{p(\cdot)}{\beta}\right)^{\prime}}^{\frac{n}{n-\alpha \beta}} \\
& \lesssim|B|^{\left(1-\frac{\alpha \beta}{n}\right) \frac{n}{n-\alpha \beta}}=|B| .
\end{aligned}
$$

Conversely, let us now suppose $w^{\frac{\beta n}{n-\alpha \beta}} \in A_{q(\cdot)\left(\frac{1}{\beta}-\frac{\alpha}{n}\right)}$. Using again (4.6) and (4.7), we obtain that

$$
\begin{aligned}
\left\|w^{\beta} \chi_{B}\right\|_{\frac{q(\cdot)}{\beta}}\left\|w^{-\beta} \chi_{B}\right\|_{\frac{p(\cdot)}{p(\cdot)-\beta}} & =\left\|w^{\frac{n \beta}{n-\alpha \beta}} \chi_{B}\right\|_{q(\cdot)\left(\frac{1}{\beta}-\frac{\alpha}{n}\right)}^{1-\frac{\alpha \beta}{n}}\left\|w^{\frac{-n \beta}{n-\alpha \beta}} \chi_{B}\right\|_{\frac{q(\cdot)(n-\alpha \beta)}{q(\cdot)(n-\alpha \beta)-n \beta}}^{1-\frac{\alpha \beta}{n}} \\
& =\left\|w^{\frac{n \beta}{n-\alpha \beta}} \chi_{B}\right\|_{q(\cdot)\left(\frac{1}{\beta}-\frac{\alpha}{n}\right)}^{1-\frac{\alpha \beta}{n}}\left\|w^{\frac{-n \beta}{n-\alpha \beta}} \chi_{B}\right\|_{\left(q(\cdot)\left(\frac{1}{\beta}-\frac{\alpha}{n}\right)\right)^{\prime}}^{1-\frac{\alpha \beta}{n}} \lesssim|B|^{1-\frac{\alpha \beta}{n}}
\end{aligned}
$$

We proceed now with the proof of (4.3). Since $0<\delta<1<q^{-}$, we have that

$$
\left\|w^{\delta} \chi_{B}\right\|_{\frac{q(\cdot)}{\delta}}\left\|w^{-\delta} \chi_{B}\right\|_{\frac{q(\cdot)}{q(\cdot)-\delta}}=\left\|w \chi_{B}\right\|_{q(\cdot)}^{\delta}\left\|w^{-\delta} \chi_{B}\right\|_{\frac{q(\cdot)}{q(\cdot)-\delta}} .
$$

If $u(\cdot)=q(\cdot) /(q(\cdot)-\delta)$, from the relation between $p(\cdot)$ and $q(\cdot)$ we have that $1 / u(\cdot)=(n-n \delta+\alpha \delta) / n+\delta / p^{\prime}(\cdot)$. Thus, by the generalized Hölder inequality and the hypothesis on $w$ we obtain that

$$
\begin{aligned}
\left\|w^{\delta} \chi_{B}\right\|_{\frac{q(\cdot)}{\delta}}\left\|w^{-\delta} \chi_{B}\right\|_{\frac{q(\cdot)}{q(\cdot)-\delta}} & \leq\left\|w \chi_{B}\right\|_{q(\cdot)}^{\delta}\left\|w^{-1} \chi_{B}\right\|_{p^{\prime}(\cdot)}^{\delta}\left\|\chi_{B}\right\|_{\frac{n}{n-n \delta+\alpha \delta}} \\
& \lesssim|B|^{\delta\left(1-\frac{\alpha}{n}\right)}|B|^{1-\delta\left(1-\frac{\alpha}{n}\right)}=|B| .
\end{aligned}
$$

In order to prove (4.4), take $r$ and $\delta$ as in the hypothesis. Since $0<\delta<1 \leq r \leq$ $q^{-}$, we have that

$$
\left\|w^{\delta} \chi_{B}\right\|_{\frac{q(\cdot)}{\delta}}\left\|w^{-\delta} \chi_{B}\right\|_{\frac{q(\cdot)}{q(\cdot)-\delta}}=\left\|w^{r} \chi_{B}\right\|_{\frac{q(\cdot)}{r}}^{\frac{\delta}{r}}\left\|w^{-\delta} \chi_{B}\right\|_{\frac{q(\cdot)}{q(\cdot)-\delta}} .
$$

Let $u(\cdot)$ be as in (4.3). From the fact that $1 / u(\cdot)=1 /\left((r / \delta)\left(\frac{q(\cdot)}{r}\right)^{\prime}\right)+(r-\delta) / r,(4.8)$ and the hypothesis on the weight, we obtain that

$$
\begin{aligned}
\left\|w^{\delta} \chi_{B}\right\|_{\frac{q(\cdot)}{\delta}}\left\|w^{-\delta} \chi_{B}\right\|_{\frac{q(\cdot)}{q(\cdot)-\delta}} & \leq\left\|w^{r} \chi_{B}\right\|_{\frac{q(\cdot)}{r}}^{\frac{\delta}{r}}\left\|w^{-r} \chi_{B}\right\|_{\left(\frac{q(\cdot)}{r}\right)^{\prime}}^{\frac{\delta}{r}}\left\|\chi_{B}\right\|_{\frac{r}{r-\delta}} \\
& \leq|B|^{\frac{\delta}{r}}|B|^{1-\frac{\delta}{r}}=|B| .
\end{aligned}
$$

The following pointwise estimate is an important tool in order to prove Theorems $2.3,2.6$ and 2.15 .

4.9. Theorem. Let $0 \leq \alpha<n$ and let $p \in \mathcal{P}^{*}\left(\mathbf{R}^{n}\right)$ such that $p^{+}<n / \alpha$. Let $q(\cdot)$ and $s(\cdot)$ be the functions defined by $1 / q(x)=1 / p(x)-\alpha / n$ and $s(x)=1+q(x) / p^{\prime}(x)$, respectively. Let $\eta$ and $\phi$ be Young functions such that $\phi^{-1}(t) t^{\alpha / n} \lesssim \eta^{-1}(t)$. Then for every measurable function $f$ and every weight $w$, the following inequality

$$
M_{\alpha, \eta}(f / w)(x) \lesssim\left(M_{\psi}\left(|f|^{p(\cdot) / s(\cdot)} w^{-q(\cdot) / s(\cdot)}\right)(x)\right)^{1-\alpha / n}\left(\int_{\mathbf{R}^{n}}|f(y)|^{p(y)} d y\right)^{\alpha / n}
$$

holds, where $\psi(t)=\phi\left(t^{1-\alpha / n}\right)$. 
4.10. Remark. For the fractional maximal operator, that is, when $\eta(t)=t$, the theorem above was proved in [21].

Proof of Theorem 4.9. Let $g(x)=|f(x)|^{p(x) / s(x)} w(x)^{-q(x) / s(x)}$ and $B=B(x, R)$, $R>0, x \in \mathbf{R}^{n}$. Then

$$
|f(x)| / w(x)=g^{s(x) / p(x)-1+\alpha / n} w(x)^{q(x) / p(x)-1} g(x)^{1-\alpha / n} .
$$

Let $x \in \mathbf{R}^{n}$ and a fixed ball $B \ni x$. By the generalized Hölder inequality and the fact that

$$
g^{(s(x) / p(x)-1+\alpha / n) n / \alpha} w(x)^{(q(x) / p(x)-1) n / \alpha}=|f(x)|^{p(x)},
$$

we get

$$
\begin{aligned}
|B|^{\alpha / n}\|f / w\|_{\eta, B} & \leq C|B|^{\alpha / n}\left\|g^{1-\alpha / n}\right\|_{\phi, B}\left\|g^{s(\cdot) / p(\cdot)+\alpha / n-1} w^{q(\cdot) / p(\cdot)-1}\right\|_{n / \alpha, B} \\
& =C\|g\|_{\psi, B}^{1-\alpha / n}|B|^{\alpha / n}\left(\frac{1}{|B|} \int_{B}|f(x)|^{p(x)}\right)^{\alpha / n} \\
& \lesssim\left[M_{\psi}(g)(x)\right]^{1-\alpha / n}\left(\int_{\mathbf{R}^{n}}|f(x)|^{p(x)}\right)^{\alpha / n} .
\end{aligned}
$$

Particularly, when $\eta(t)=t^{\beta}\left(1+\log ^{+} t\right)^{\gamma}$, with $1 \leq \beta<n / \alpha$ and $\gamma \geq 0$, Theorem 4.9 holds with $\psi(t) \approx t^{\frac{\beta(n-\alpha)}{n-\alpha \beta}}\left(1+\log ^{+} t\right)^{\frac{n \gamma}{n-\alpha \beta}}$. In fact, if $\phi(t)=t^{\frac{n \beta}{n-\alpha \beta}}(1+$ $\left.\log ^{+} t\right)^{\frac{n \gamma}{n-\alpha \beta}}$ then

$$
\phi^{-1}(t) t^{\frac{\alpha}{n}} \approx \frac{t^{1 / \beta-\alpha / n}}{\left(1+\log ^{+} t\right)^{\gamma / \beta}} t^{\frac{\alpha}{n}} \approx \eta^{-1}(t)
$$

and thus $\psi(t)=\phi\left(t^{1-\alpha / n}\right)=t^{\frac{n \beta}{n-\alpha \beta} \frac{n-\alpha}{n}}\left(1+\log ^{+}\left(t^{1-\alpha / n}\right)\right)^{\gamma n /(n-\alpha \beta)} \approx t^{\frac{\beta(n-\alpha)}{n-\alpha \beta}}(1+$ $\left.\log ^{+} t\right)^{\frac{n \gamma}{n-\alpha \beta}}$.

We now prove a pointwise inequality which is crucial in the proof of Theorem 2.11, in the spirit of the results obtained for the Hardy-Littlewood maximal operator by Diening in [15] for certain exponents $p$, and by Capone, Cruz-Uribe and Fiorenza in [5] for more general exponents. In order to prove it, we will use the following lemma which is an extension of Lemma 2.5 of [5] involving Young averages.

4.11. Lemma. Given a set $A \subset \mathbf{R}^{n}$ with $0<|A|<\infty$ and two non-negative functions $r(\cdot)$ and $s(\cdot)$, suppose that for each $y \in A$,

$$
0 \leq s(y)-r(y) \leq \frac{C}{\log (e+|z(y)|)}
$$

where $z: A \rightarrow \mathbf{R}^{n}$. Then, for every measurable set $D$ and every $t>0$ there exists a positive constant $C_{t}$ such that for every function $f$,

$$
\left\||f(\cdot)|^{r(\cdot)} \chi_{D}\right\|_{\eta, A} \leq 2 C_{t}\left\||f(\cdot)|^{s(\cdot)} \chi_{D}\right\|_{\eta, A}+2\left\|S_{t}(z(\cdot))^{r_{A}^{-}} \chi_{D}\right\|_{\eta, A},
$$

where $S_{t}(x)=(e+|x|)^{-t n}$.

Proof. Let $\lambda=2 C_{t}\left\|f^{s} \chi_{D}\right\|_{\eta, A}+2\left\|S_{t}(z)^{r_{A}^{-}} \chi_{D}\right\|_{\eta, A}>0$. Without loss of generality, we will suppose that $|D \cap A|>0$ and $f(x) \neq 0$ in almost every $x \in D \cap A$; otherwise, there is nothing to prove. 
Let us define $D_{1}=\left\{x \in A \cap D:|f(x)| \geq S_{t}(z(x))\right\}$. Notice first that from the hypothesis over $s(\cdot)$ and $r(\cdot)$ we have that for every $x \in D_{1}$

$$
\begin{aligned}
|f(x)|^{r(x)} & =|f(x)|^{s(x)}|f(x)|^{r(x)-s(x)} \leq|f(x)|^{s(x)}\left(S_{t}(z(x))\right)^{r(x)-s(x)} \\
& \leq|f(x)|^{s(x)}(e+|z(x)|)^{\operatorname{tnC} / \log (e+|z(x)|)}=C_{t}|f(x)|^{s(x)} .
\end{aligned}
$$

On the other hand, for $x \in(A \cap D) \backslash D_{1}$, since $S_{t}(z(x)) \leq 1$, we have that

$$
|f(x)|^{r(x)} \leq\left(S_{t}(z(x))\right)^{r(x)} \leq\left(S_{t}(z(x))\right)^{r_{A}^{-}} .
$$

Then, using both (4.12) and (4.13) we obtain

$$
\begin{aligned}
& \frac{1}{|A|} \int_{A} \eta\left(\frac{|f(x)|^{r(x)} \chi_{D}(x)}{\lambda}\right) d x \\
& \leq \frac{1}{|A|} \int_{D_{1}} \eta\left(\frac{|f(x)|^{r(x)}}{2 C_{t}\left\|f^{s} \chi_{D}\right\|_{\eta, A}}\right) d x+\frac{1}{|A|} \int_{(A \cap D) \backslash D_{1}} \eta\left(\frac{|f(x)|^{r(x)}}{2\left\|S_{t}(z(\cdot))^{r_{A}^{-}} \chi_{D}\right\|_{\eta, A}}\right) d x \\
& \leq \frac{1}{|A|} \int_{D_{1}} \eta\left(\frac{|f(x)|^{s(x)}}{2\left\|f^{s} \chi_{D}\right\|_{\eta, A}}\right) d x+\frac{1}{|A|} \int_{(A \cap D) \backslash D_{1}} \eta\left(\frac{\left(S_{t}(z(x))\right)^{r}}{2\left\|S_{t}^{-}(z(\cdot))^{r_{A}^{-}} \chi_{D}\right\|_{\eta, A}}\right) d x \\
& \leq \frac{1}{2} \frac{1}{|A|} \int_{A} \eta\left(\frac{|f(x)|^{s(x)} \chi_{D}(x)}{\left\|f^{s} \chi_{D}\right\|_{\eta, A}}\right) d x+\frac{1}{2} \frac{1}{|A|} \int_{A} \eta\left(\frac{\left(S_{t}(z(x))\right)^{r_{A}^{-}} \chi_{D}(x)}{\left\|S_{t}(z(\cdot))^{r_{A}^{-}} \chi_{D}\right\|_{\eta, A}}\right) d x \\
& \leq \frac{1}{2}+\frac{1}{2}=1,
\end{aligned}
$$

and thus,

$$
\left\||f(\cdot)|^{r(\cdot)} \chi_{D}\right\|_{\eta, A} \leq 2 C_{t}\left\||f(\cdot)|^{s(\cdot)} \chi_{D}\right\|_{\eta, A}+2\left\|S_{t}(z(\cdot))^{r_{A}^{-}} \chi_{D}\right\|_{\eta, A} \cdot
$$

4.14. Remark. Particularly, under the same hypothesis on the functions $r(\cdot)$ and $s(\cdot)$ and by taking $\eta(t)=t$ we obtain the following inequality, proved in [5].

$$
\frac{1}{|A|} \int_{A \cap D}|f(x)|^{r(x)} d x \leq \frac{2 C_{t}}{|A|} \int_{A \cap D}|f(x)|^{s(x)} d x+\frac{2}{|A|} \int_{A \cap D} S_{t}(z(x))^{r_{A}^{-}} d x .
$$

We also give the next useful lemma proved by Diening in [15]. The result relates the local log-Hölder condition and the size of the balls.

4.16. Lemma. An exponent $p \in \mathcal{P}^{*}\left(\mathbf{R}^{n}\right)$ satisfies condition (1.3) if and only if there exists a positive constant $C$ such that, for every ball $B,|B|^{p_{B}^{-}-p_{B}^{+}} \leq C$.

We are now in position to give the pointwise estimate. It should be mentioned that a different proof of (4.18) is given in [23].

4.17. Theorem. Let $p \in \mathcal{P}^{\log }\left(\mathbf{R}^{n}\right)$ and $\eta$ a Young function. Let $S: \mathbf{R}^{n} \rightarrow \mathbf{R}$ be the function defined by $S(x)=(e+|x|)^{-n}$.

(4.18) If $p^{-}>1$ and $\eta \in B_{p^{-}}$, then the inequality

$$
\left(M_{\eta} f(x)\right)^{p(x)} \lesssim M\left(|f(\cdot)|^{p(\cdot)}\right)(x)+S(x)^{p^{-}}
$$

holds for every function $f$ such that $\left\|f \chi_{\{|f|>1\}}\right\|_{p(\cdot)} \leq 1$;

(4.19) If $p^{-}=1$ and $\eta \in B_{q}$ for every $q>1$, then the inequality

$$
\left(M_{\eta} f(x)\right)^{p(x)} \lesssim M_{\eta}\left(|f(\cdot)|^{p(\cdot)}\right)(x)+S(x)
$$

holds for every function $f$ such that $\int_{\{|f| \geq 1\}} \eta\left(|f(x)|^{p(x)}\right) d x \leq 1$. 
Proof. In order to prove (4.18) let us first consider $p^{-}>1$ and $\eta \in B_{p^{-}}$. Then, it is enough to show that $\|f\|_{\eta, B}^{p(x)} \lesssim M\left(|f(\cdot)|^{p(\cdot)}\right)(x)+S(x)^{p^{-}}$for every ball $B=$ $B\left(x_{0}, R\right) \ni x$. From the facts that $p_{B}^{-} \geq p^{-}>1$ and the $B_{p}$ classes are increasing we obtain that $\eta \in B_{p_{B}^{-}}$. Thus, an easy consequence of the definition of the $B_{p_{B}^{-}}$class is that $\eta(t) \lesssim t^{p_{B}^{-}}$for $t>t_{0}$. Particularly, this implies that $\left\|f_{1}\right\|_{\eta, B} \lesssim\left\|f_{1}\right\|_{p_{B}^{-}, B}$.

Fix $B \ni x$ and let $f_{1}=f \chi_{\{|f|>1\}}$ and $f_{2}=f-f_{1}$. Let us first estimate $\left\|f_{1}\right\|_{\eta, B}^{p(x)}$. Since $\left\|f_{1}\right\|_{p(\cdot)} \leq 1$ we have that

$$
\begin{aligned}
\left\|f_{1}\right\|_{\eta, B}^{p(x)} & \lesssim\left(\frac{1}{|B|} \int_{B}\left|f_{1}(y)\right|^{p_{B}^{-}} d y\right)^{\frac{p(x)}{p_{B}^{-}}} \leq\left(\frac{1}{|B|} \int_{B}\left|f_{1}(y)\right|^{p(y)} d y\right)^{\frac{p(x)}{p_{B}^{-}}} \\
& =\left(\frac{1}{|B|} \int_{B}\left|f_{1}(y)\right|^{p(y)} d y\right)^{\frac{p(x)}{p_{B}^{-}}-1}\left(\frac{1}{|B|} \int_{B}|f(y)|^{p(y)} d y\right) \\
& \leq|B|^{\frac{p_{B}^{-}-p(x)}{p_{B}^{-}}} M\left(|f(\cdot)|^{p(\cdot)}\right)(x) .
\end{aligned}
$$

If $|B|>1$, since the exponent $\left(p_{B}^{-}-p(x)\right) / p_{B}^{-} \leq 0$, we have $|B|^{\left(p_{B}^{-}-p(x)\right) / p_{B}^{-}} \leq 1$. On the other hand, if $|B| \leq 1$, from the hypothesis on $p$ and by applying Lemma 4.16 we have that $|B|^{\left(p_{B}^{-}-p(x)\right) / p_{B}^{-}} \leq|B|^{p_{B}^{-}-p_{B}^{+}} \leq C$. In both cases, $\left\|f_{1}\right\|_{\eta, B}^{p(x)} \lesssim M\left(|f(\cdot)|^{p(\cdot)}\right)(x)$.

Let us now estimate $\left\|f_{2}\right\|_{\eta, B}^{p(x)}$. Consider the sets $E=B \cap B(0,|x|)$ and $F=B \backslash$ $B(0,|x|)$. We split $f_{2}=f_{2}^{E}+f_{2}^{F}=f_{2} \chi_{E}+f_{2} \chi_{F}$. Then, $\left\|f_{2}\right\|_{\eta, B}^{p(x)} \lesssim\left\|f_{2}^{E}\right\|_{\eta, B}^{p(x)}+\left\|f_{2}^{F}\right\|_{\eta, B}^{p(x)}$.

In order to estimate $\left\|f_{2}^{E}\right\|_{\eta, B}^{p(x)}$, we will use again the fact that $\eta \in B_{p_{B}^{-}}$. From the decay $\log$-Hölder condition over $p$ we have that for every $y \in E$

$$
0 \leq p(y)-p_{B}^{-} \leq \frac{C}{\log (e+|y|)}
$$

Thus, we can apply $(4.15)$ with $r(\cdot)$ and $s(\cdot)$ replaced by $p_{B}^{-}$and $p(\cdot)$ respectively, $z(y)=y$ and $t=1$ to obtain

$$
\begin{aligned}
\left\|f_{2}^{E}\right\|_{\eta, B}^{p(x)} & \lesssim\left(\frac{1}{|B|} \int_{B}\left|f_{2}^{E}(y)\right|^{p_{B}^{-}} d y\right)^{\frac{p(x)}{p_{B}^{-}}} \leq \frac{1}{|B|} \int_{B}\left|f_{2}^{E}(y)\right|^{p_{B}^{-}} d y \\
& \lesssim \frac{1}{|B|} \int_{B}\left|f_{2}^{E}(y)\right|^{p(y)} d y+\frac{1}{|B|} \int_{E}(e+|y|)^{-n p_{B}^{-}} d y \\
& \leq \frac{1}{|B|} \int_{B}|f(y)|^{p(y)} d y+\frac{1}{|B|} \int_{E}(e+|y|)^{-n p_{B}^{-}} d y \\
& \leq M\left(|f(\cdot)|^{p(\cdot)}\right)(x)+\frac{1}{|B|} \int_{E}(e+|y|)^{-n p_{B}^{-}} d y .
\end{aligned}
$$

If $R<|x| / 4$, it is easy to see that $|y| \approx|x|$ for every $y \in E$ so

$$
\frac{1}{|B|} \int_{E}(e+|y|)^{-n p_{B}^{-}} d y \lesssim \frac{1}{|B|} \int_{E}(e+|x|)^{-n p_{B}^{-}} \leq(e+|x|)^{-n p_{B}^{-}} \leq S(x)^{p^{-}} .
$$

If $R \geq|x| / 4$ and $|x|<1$, we have that $e+|x| \lesssim e+|y|$ so we proceed as in (4.18). 
Finally, if $R \geq|x| / 4$ and $|x| \geq 1$, then $4^{n}|B| \geq|B(0,|x|)|$ and thus

$$
\begin{aligned}
& \frac{1}{|B|} \int_{E}(e+|y|)^{-n p_{B}^{-}} d y \lesssim \frac{1}{|B(0,|x|)|} \int_{B(0,|x|)}(e+|y|)^{-n p_{B}^{-}} d y \\
& \lesssim|x|^{-n} \int_{B(0,|x|)}(e+|y|)^{-n p_{B}^{-}} d y \lesssim(e+|x|)^{-n} \int_{B(0,|x|)}(e+|y|)^{-n p_{B}^{-}} d y .
\end{aligned}
$$

Since

$$
\int_{B(0,|x|)}(e+|y|)^{-n p_{B}^{-}} d y \leq \int_{e}^{e+|x|} \rho^{n-1-n p_{B}^{-}} d \rho \leq(e+|x|)^{n-n p_{B}^{-}}
$$

we get

$$
\frac{1}{|B|} \int_{E}(e+|y|)^{-n p_{B}^{-}} d y \lesssim(e+|x|)^{-n p_{B}^{-}} \leq S(x)^{p^{-}} .
$$

Thus, we conclude the estimate for $f_{2}^{E}$.

By noticing that $|y| \geq|x|$ for all $y \in F$, we can use Lemma 4.11 with $p_{B}^{-}, p(\cdot)$, $z(y)=x$ and $t=1$ to obtain

$$
\begin{aligned}
\left\|f_{2}^{F}\right\|_{\eta, B}^{p(x)} & \lesssim\left(\frac{1}{|B|} \int_{B}\left|f_{2}^{F}(y)\right|^{p_{B}^{-}} d y\right)^{\frac{p(x)}{p_{B}^{-}}} \leq \frac{1}{|B|} \int_{B}\left|f_{2}^{F}(y)\right|^{p_{B}^{-}} d y \\
& \lesssim \frac{1}{|B|} \int_{B}|f(y)|^{p(y)} d y+\frac{1}{|B|} \int_{F}(e+|x|)^{-n p^{-}} d y \\
& \leq M\left(|f(\cdot)|^{p(\cdot)}\right)(x)+S(x)^{p^{-}}
\end{aligned}
$$

Therefore, combining all the cases we get $\|f\|_{\eta, B}^{p(x)} \lesssim M\left(|f(\cdot)|^{p(\cdot)}\right)(x)+S(x)^{p^{-}}$for every ball $B \ni x$.

In order to prove (4.19), let us consider $p^{-}=1$ and $\eta \in B_{q}$ for all $q>1$. Then, it is enough to show that $\|f\|_{\eta, B}^{p(x)} \lesssim M_{\eta}\left(|f(\cdot)|^{p(\cdot)}\right)(x)+S(x)$ for every ball $B \ni x$. Given a ball $B \ni x$, if $p_{B}^{-}>1$, we proceed as in (4.18) because the hypothesis over $f$ implies $\left\|f_{1}\right\|_{p(\cdot)} \leq 1$. Suppose that $p_{B}^{-}=1$. We split $f=f_{1}+f_{2}$ as before so $\|f\|_{\eta, B}^{p(x)} \lesssim\left\|f_{1}\right\|_{\eta, B}^{p(x)}+\left\|f_{2}\right\|_{\eta, B}^{p(x)}$.

First, we will estimate $\left\|f_{1}\right\|_{\eta, B}^{p(x)}$. If $|B| \leq 1$, by the convexity of $\eta$ we have that

$$
\frac{1}{|B|} \int_{B} \eta\left(\left|B \| f_{1}(y)\right|^{p(y)}\right) d y \leq \int_{\{|f|>1\}} \eta\left(|f(y)|^{p(y)}\right) d y \leq 1
$$

and thus $\left\|\left|B\left\|\left.f_{1}\right|^{p}\right\|_{\eta, B} \leq 1\right.\right.$. Then, by Lemma 4.16 we obtain that

$$
\begin{aligned}
\left\|f_{1}\right\|_{\eta, B}^{p(x)} & \leq|B|^{-p(x)}\left\|\left.\left|B\left\|\left.f_{1}\right|^{p}\right\|_{\eta, B}^{p(x)} \leq\right| B\right|^{1-p(x)}\right\|\left|f_{1}\right|^{p}\left\|_{\eta, B}=|B|^{p_{B}^{-}-p(x)}\right\|\left|f_{1}\right|^{p} \|_{\eta, B} \\
& \leq|B|^{p_{B}^{-}-p_{B}^{+}}\left\||f|^{p}\right\|_{\eta, B} \leq C M_{\eta}\left(|f(\cdot)|^{p(\cdot)}\right)(x) .
\end{aligned}
$$

If $|B|>1$, from the hypothesis over $f$ we get

$$
\frac{1}{|B|} \int_{B} \eta\left(\left|f_{1}(y)\right|\right) d y \leq \int_{\{|f|>1\}} \eta\left(|f(y)|^{p(y)}\right) d y \leq 1 .
$$

Hence,

$$
\left\|f_{1}\right\|_{\eta, B}^{p(x)} \leq\left\|f_{1}\right\|_{\eta, B} \leq\left\|f^{p}\right\|_{\eta, B} \leq M_{\eta}\left(|f(\cdot)|^{p(\cdot)}\right)(x)
$$


Now, we estimate $\left\|f_{2}\right\|_{\eta, B}^{p(x)}$, spliting it as before into $f_{2}^{E}$ and $f_{2}^{F}$. Using Lemma 4.11 with $p(\cdot), p_{B}^{-}=1$ and $t=1$, we obtain

$\left\|f_{2}^{E}\right\|_{\eta, B}^{p(x)} \leq\left\|f_{2}^{E}\right\|_{\eta, B}=\left\|f_{2} \chi_{E}\right\|_{\eta, B} \lesssim\left\|f_{2}^{p} \chi_{E}\right\|_{\eta, B}+\left\|S \chi_{E}\right\|_{\eta, B} \leq M_{\eta}\left(|f(\cdot)|^{p(\cdot)}\right)+\left\|S \chi_{E}\right\|_{\eta, B}$,

We will show that $\left\|S \chi_{E}\right\|_{\eta, B} \leq S(x)$.

Recall that if $R<|x| / 4$ or if $R \geq|x| / 4$ and $|x| \leq 1$, we had that $S(y) \lesssim S(x)$. Thus, in both cases, $\left\|S \chi_{E}\right\|_{\eta, B} \approx\left\|S(x) \chi_{E}\right\|_{\eta, B} \leq S(x)$.

Now, if $R \geq|x| / 4$ and $|x|>1$ we know that $4^{n}|B| \geq|B(0,|x|)|,|x|^{-n} \lesssim S(x)<$ $S(y)$. Fix $q>1$ so $\eta \in B_{q}$. Let $c>1$ be a constant to be determined. Then,

$$
\begin{aligned}
\frac{1}{|B|} \int_{B} \eta\left(\frac{S(y) \chi_{E}(y)}{c S(x)}\right) d y & \leq \frac{1}{|B|} \int_{E} \frac{1}{c} \eta\left(\frac{S(y)}{S(x)}\right) d y \leq \frac{1}{|B|} \int_{E} \frac{1}{c}\left(\frac{S(y)}{S(x)}\right)^{q} d y \\
& \leq \frac{C}{c} \frac{1}{|x|^{n}}(e+|x|)^{n q} \int_{B(0,|x|)} \frac{1}{(e+|y|)^{n q}} d y \\
& \leq \frac{C}{c} \frac{(e+|x|)^{n q}}{|x|^{n}}(e+|x|)^{n-n q} \leq 1
\end{aligned}
$$

by taking $c$ conveniently. Therefore, $\left\|S \chi_{E}\right\|_{\eta, B} \lesssim S(x)$.

Finally, since $|y| \geq|x|$ for all $y \in F$, we use Lemma 4.11 with $p_{B}^{-}, p(\cdot), z(y)=x$ and $t=1$ to get

$$
\begin{aligned}
\left\|f_{2}^{F}\right\|_{\eta, B}^{p(x)} & \lesssim\left\|f_{2}^{p} \chi_{F}\right\|_{\eta, B}+\left\|S \chi_{E}\right\|_{\eta, B} \leq M_{\eta}\left(|f(\cdot)|^{p(\cdot)}\right)+\left\|S(x) \chi_{E}\right\|_{\eta, B} \\
& =M_{\eta}\left(|f(\cdot)|^{p(\cdot)}\right)+S(x) .
\end{aligned}
$$

In the following examples we give some Young functions satisfying the conditions of Theorem 4.17 and the corresponding pointwise inequalities.

i) If $p^{-}>1,1 \leq \beta<p^{-}$and $\gamma \geq 0$ it is easy to see that the functions $\eta(t)=$ $t^{\beta}\left(1+\log ^{+} t\right)^{\gamma}$ and $\xi(t)=t^{\beta}\left(1+\log \left(1+\log ^{+} t\right)\right)^{\gamma}$ belong to the $B_{p^{-}}$class. Then, by Theorem 4.17 we obtain the following inequalities

$$
\left(M_{L^{\beta}(\log L)^{\gamma}} f(x)\right)^{p(x)} \lesssim M\left(|f(\cdot)|^{p(\cdot)}\right)+S(x)^{p^{-}},
$$

and

$$
\left(M_{L^{\beta}(\log (\log L))^{\gamma}} f(x)\right)^{p(x)} \lesssim M\left(|f(\cdot)|^{p(\cdot)}\right)+S(x)^{p^{-}} .
$$

ii) If $\beta=1$ and $\gamma \geq 0$, both functions $\eta(t)=t\left(1+\log ^{+} t\right)^{\gamma}$ and $\xi(t)=t(1+$ $\left.\log \left(1+\log ^{+} t\right)\right)^{\gamma}$ satisfy the $B_{q}$ condition for every $q>1$. Thus, if $p^{-}=1$ we obtain that

$$
\left(M_{L(\log (\log L))^{\gamma}} f(x)\right)^{p(x)} \lesssim M_{L(\log (\log L))^{\gamma}}\left(|f(\cdot)|^{p(\cdot)}\right)+S(x), \quad \text { if } p^{-}=1
$$

and

$$
\left(M_{L(\log (\log L))^{\gamma}} f(x)\right)^{p(x)} \lesssim M\left(|f(\cdot)|^{p(\cdot)}\right)+S(x)^{p^{-}}, \quad \text { if } p^{-}>1 .
$$

The following weak-type inequality was established in [27] and it allows us to prove Theorem 2.11.

4.19. Lemma. Let $\eta$ be a Young function and $w$ a weight. Then, the following estimate holds

$$
w\left(\left\{x \in \mathbf{R}^{n}: M_{\eta} f(x)>2 \lambda\right\}\right) \lesssim \int_{\{|f|>\lambda\}} \eta\left(\frac{|f(x)|}{\lambda}\right) M w(x) d x
$$

for every $\lambda>0$. 


\section{Proofs of the main results}

We shall first introduce some notation and definitions needed in the next proof. Given $p \in \mathcal{P}^{*}\left(\mathbf{R}^{n}\right)$ and a weight $w$, let $\phi_{p(\cdot), w}(x, t)=(t w(x))^{p(x)}$. The authors in [9] defined the following classes of functions over cubes instead of balls, but it is easy to see that we can make such a change and all the results still hold.

5.1. Definition. Given $p \in \mathcal{P}^{*}\left(\mathbf{R}^{n}\right)$ and a weight $w$, the function $\phi_{p(\cdot), w} \in \mathcal{A}$ if the inequality

$$
\left\|w \sum_{B \in \mathcal{B}} \chi_{B} \frac{1}{|B|} \int_{B}|f|\right\|_{p(\cdot)} \lesssim\|w f\|_{p(\cdot)}
$$

holds for every family $\mathcal{B}$ of disjoint balls $B$ and every $f \in L_{w}^{p(\cdot)}\left(\mathbf{R}^{n}\right)$.

It is easy to see that $\phi_{p(\cdot), w} \in \mathcal{A}$ implies $w \in A_{p(\cdot)}$. Moreover, the authors in [17] showed that the converse also holds, by additionally assuming that $p \in \mathcal{P}^{\log }\left(\mathbf{R}^{n}\right)$.

Proof of Theorem 2.1. Let us first suppose that $w^{\beta} \in A_{\frac{p(\cdot)}{\beta}}$. Since $p \in \mathcal{P}^{\log }\left(\mathbf{R}^{n}\right)$, so is $p / \beta$. Then, as we recently observe, we have that $\varphi_{\frac{p(\cdot)}{\beta}, w^{\beta}} \in \mathcal{A}$. From the fact that the class $\mathcal{A}$ is left open (see Corollary 5.4 .15 of [17]), there exists $0<\epsilon<p^{-}-\beta$ such that $\varphi_{\frac{p(\cdot)}{\beta+\epsilon}, w^{\beta+\epsilon}} \in \mathcal{A}$. This implies that $w^{\beta+\epsilon} \in A_{\frac{p(\cdot)}{\beta+\epsilon}}$.

Now, for this $\epsilon$, we have that $M_{\eta}(f) \lesssim M_{\beta+\epsilon}(f)=M\left(f^{\beta+\epsilon}\right)^{\frac{1}{\beta+\epsilon}}$. Then, given $f \in L_{w}^{p(\cdot)}\left(\mathbf{R}^{n}\right)$

$$
\left\|w M_{\eta} f\right\|_{p(\cdot)} \lesssim\left\|w M_{\beta+\epsilon} f\right\|_{p(\cdot)}=\left\|w^{\beta+\epsilon} M\left(f^{\beta+\epsilon}\right)\right\|_{\frac{p(\cdot)}{\beta+\epsilon}}^{\frac{1}{\beta+\epsilon}}
$$

From the hypothesis on $f$ we have that $f^{\beta+\epsilon} \in L_{w^{\beta+\epsilon}}^{\frac{p(\cdot)}{\beta+\epsilon}}\left(\mathbf{R}^{n}\right)$, and thus, from the fact that $M$ is bounded on $L_{w^{\beta+\epsilon}}^{\frac{p(\cdot)}{\beta+\epsilon}}\left(\mathbf{R}^{n}\right)$ for $w^{\beta+\epsilon} \in A_{\frac{p(\cdot)}{\beta+\epsilon}}$, we get the desired result.

Conversely, if $M_{\eta}$ is bounded on $L_{w}^{p(\cdot)}\left(\mathbf{R}^{n}\right)$, since $M_{\beta}(f) \lesssim M_{\eta}$, we have that

$$
\left\|w^{\beta} M\left(f^{\beta}\right)\right\|_{\frac{p(\cdot)}{\beta}}^{\frac{1}{\beta}}=\left\|w M_{\beta}(f)\right\|_{p(\cdot)} \lesssim\left\|w M_{\eta} f\right\|_{p(\cdot)} \lesssim\|w f\|_{p(\cdot)}=\left\|w^{\beta} f^{\beta}\right\|_{\frac{p(\cdot)}{\beta}}^{\frac{1}{\beta}} .
$$

The condition $w^{\beta} \in A_{\frac{p(\cdot)}{\beta}}$ follows immediately from the boundedness properties of $M$.

Proof of Theorem 2.2. We will only prove that $w \in A_{p(\cdot), q(\cdot)}$ is a necessary condition for the boundedness of $M_{\alpha}$. The sufficient condition will be proved in Theorem 2.6 in a more general way.

Let us suppose that $M_{\alpha}$ is bounded from $L_{w}^{p(\cdot)}\left(\mathbf{R}^{n}\right)$ into $L_{w}^{q(\cdot)}\left(\mathbf{R}^{n}\right)$, for $p$ and $q$ as in the hypothesis. By applying Lemma 3.4, we obtain

$$
\begin{aligned}
\left\|w \chi_{B}\right\|_{q(\cdot)}\left\|w^{-1} \chi_{B}\right\|_{p^{\prime}(\cdot)} & \leq 2\left\|w \chi_{B}\right\|_{q(\cdot)} \sup _{\|g\|_{p(\cdot)} \leq 1} \int_{B}|g(x)| w^{-1}(x) d x \\
& =2 \sup _{\|g\|_{p(\cdot)} \leq 1}\left\|w \chi_{B} \int_{B}|g| w^{-1}\right\|_{q(\cdot)} \\
& =2|B|^{1-\frac{\alpha}{n}} \sup _{\|g\|_{p(\cdot)} \leq 1}\left\|w \chi_{B} \frac{1}{|B|^{1-\frac{\alpha}{n}}} \int_{B}|g| w^{-1}\right\|_{q(\cdot)}
\end{aligned}
$$




$$
\begin{aligned}
& \leq 2|B|^{1-\frac{\alpha}{n}} \sup _{\|g\|_{p(\cdot)} \leq 1}\left\|w M_{\alpha}\left(g w^{-1}\right)\right\|_{q(\cdot)} \\
& \lesssim|B|^{1-\frac{\alpha}{n}} \sup _{\|g\|_{p(\cdot)} \leq 1}\|g\|_{p(\cdot)} \leq|B|^{1-\frac{\alpha}{n}}
\end{aligned}
$$

which implies that $w \in A_{p(\cdot), q(\cdot)}$.

Proof of Theorem 2.3. Let us first consider $w^{\beta} \in A_{\frac{p(\cdot)}{\beta}, \frac{q(\cdot)}{\beta}}$. We want to show that the inequality $\left\|w M_{\alpha, \eta}(f)\right\|_{q(\cdot)} \lesssim\|w f\|_{p(\cdot)}$ holds for every $f \in L_{w}^{p(\cdot)}\left(\mathbf{R}^{n}\right)$, which is equivalent to prove that the inequality $\left\|w M_{\alpha, \eta}(f / w)\right\|_{q(\cdot)} \lesssim\|f\|_{p(\cdot)}$ holds for every $f \in L^{p(\cdot)}\left(\mathbf{R}^{n}\right)$.

Let us take $f \in L^{p(\cdot)}\left(\mathbf{R}^{n}\right)$. By homogeneity, we can suppose $\|f\|_{p(\cdot)}=1$.

Since $\eta^{-1}(t) \approx \frac{t^{\frac{1}{\beta}-\frac{\alpha}{n}}}{\left(1+\log ^{+} t\right)^{\frac{\gamma}{\beta}}} t^{\frac{\alpha}{n}}$, from Theorem 4.9, we have that

$$
M_{\alpha, \eta}(f / w) \lesssim\left(M_{\psi}(g)\right)^{1-\frac{\alpha}{n}}
$$

where $g(x)=|f(x)|^{p(x) / s(x)} w^{-q(x) / s(x)}, s(x)=q(x)(1-\alpha / n)$ and

$$
\psi(t) \approx t^{\beta(n-\alpha) /(n-\alpha \beta)}\left(1+\log ^{+} t\right)^{\gamma n /(n-\alpha \beta)} .
$$

Let $\tau=\frac{\beta(n-\alpha)}{n-\alpha \beta}$. Since $1 \leq \beta<p^{-}$we obtain that $1 \leq \tau<\frac{p^{-}(n-\alpha)}{n-\alpha p^{-}}=s^{-}$, where $s(\cdot)$ is the exponent defined above. From $(4.2), w^{\beta} \in A_{\frac{p(\cdot)}{\beta}, \frac{q(\cdot)}{\beta}}$ is equivalent to $\left(w^{\frac{n}{n-\alpha}}\right)^{\tau} \in A_{\frac{s(\cdot)}{\tau}}$. Also, from the fact that

$$
\int_{\mathbf{R}^{n}} g(x)^{s(x)} w(x)^{\frac{s(x) n}{n-\alpha}} d x=\int_{\mathbf{R}^{n}}|f(x)|^{p(x)} w(x)^{-q(x)} w(x)^{q(x)} d x \leq 1,
$$

we have that $g \in L_{w^{\frac{n}{n-\alpha}}}^{s(\cdot)}\left(\mathbf{R}^{n}\right)$ and $\left\|g w^{\frac{n}{n-\alpha}}\right\|_{s(\cdot)} \leq 1$. Then, we can apply Theorem 2.1 to $\psi$ and $g$ to get

$$
\begin{aligned}
\left\|w M_{\alpha, \eta}(f / w)\right\|_{q(\cdot)} & \lesssim\left\|w\left(M_{\psi} g\right)^{1-\frac{\alpha}{n}}\right\|_{q(\cdot)}=\left\|w^{\frac{n}{n-\alpha}} M_{\psi} g\right\|_{s(\cdot)}^{1-\frac{\alpha}{n}} \\
& \lesssim\left\|w^{\frac{n}{n-\alpha}} g\right\|_{s(\cdot)}^{1-\frac{\alpha}{n}} \leq 1=\|f\|_{p(\cdot)} .
\end{aligned}
$$

Observe that, particularly, when $\beta=1$ and $\gamma=0$, the proof above gives the sufficiency of Theorem 2.2.

Let us now suppose that for every $f \in L_{w}^{p(\cdot)}\left(\mathbf{R}^{n}\right)$, we have that

$$
\left\|w M_{\alpha, \eta}(f)\right\|_{q(\cdot)} \lesssim\|w f\|_{p(\cdot)} \text {. }
$$

Since $t^{\beta} \leq \eta(t)$ for every $t>0$, then $M_{\alpha, t^{\beta}} \leq M_{\alpha, \eta}$, and thus

$$
\left\|w^{\beta} f^{\beta}\right\|_{\frac{p(\cdot)}{\beta}}^{\frac{1}{\beta}}=\|w f\|_{p(\cdot)} \gtrsim\left\|w M_{\alpha, t^{\beta}}(f)\right\|_{q(\cdot)}=\left\|w^{\beta} M_{\alpha \beta}\left(f^{\beta}\right)\right\|_{\frac{q(\cdot)}{\beta}}^{\frac{1}{\beta}} .
$$

From the boundedness results of the fractional maximal operator (Theorem 2.2), we

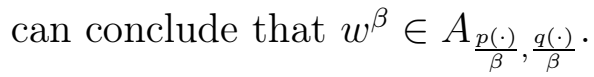

Proof of Theorem 2.5. As in the proof of Theorem 2.1, we know that there exists $\beta<r<p^{-}$such that $w^{r} \in A_{\frac{p(\cdot)}{r}}$. Since $\eta \in B_{\rho}$ for all $\rho>\beta$, by taking $\rho=r$ we 
know that $M_{\eta} \lesssim M_{r}$. Then,

$$
\left\|w M_{\eta} f\right\|_{p(\cdot)} \lesssim\left\|w M_{r} f\right\|_{p(\cdot)}=\left\|w^{r} M\left(f^{r}\right)\right\|_{\frac{p(\cdot)}{r}}^{\frac{1}{r}} .
$$

On the other hand, if $f \in L_{w}^{p(\cdot)}\left(\mathbf{R}^{n}\right)$, then $f^{r} \in L_{w^{r}}^{\frac{p(\cdot)}{r}}\left(\mathbf{R}^{n}\right)$ and by the boundedness properties of $M$, we obtain that

$$
\left\|w M_{\eta} f\right\|_{p(\cdot)} \lesssim\left\|w^{r} f^{r}\right\|_{\frac{p(\cdot)}{r}}^{\frac{1}{r}}=\|w f\|_{p(\cdot)} .
$$

Proof of Theorem 2.6. As in the proof of Theorem 2.3, we will show that

$$
\left\|w M_{\alpha, \eta}(f / w)\right\|_{q(\cdot)} \lesssim\|f\|_{p(\cdot)}
$$

for every $f \in L^{p(\cdot)}\left(\mathbf{R}^{n}\right)$ with $\|f\|_{p(\cdot)}=1$.

Under the assumptions over $\eta$, Theorem 4.9 gives us the following inequality

$$
M_{\alpha, \eta}(f / w) \lesssim\left(M_{\psi}(g)\right)^{1-\frac{\alpha}{n}}
$$

where $g(x)=|f(x)|^{p(x) / s(x)} w^{-q(x) / s(x)}, s(x)=q(x)(1-\alpha / n)$ and $\psi(t)=\phi\left(t^{1-\alpha / n}\right)$.

Since $\eta^{1+\frac{\rho \alpha}{n-\alpha}} \in B_{\frac{\rho n}{n-\alpha}}$ for all $\rho>\tau:=\beta(n-\alpha) /(n-\alpha \beta)$, we get that $\psi \in B_{\rho}$ for all $\rho>\tau$. In fact,

$$
\int_{c_{0}}^{\infty} \frac{\psi(v)}{v^{\rho}} \frac{d v}{v}=\int_{c_{0}}^{\infty} \frac{\phi\left(v^{1-\frac{\alpha}{n}}\right)}{v^{\rho}} \frac{d v}{v} \approx \int_{c_{1}}^{\infty} \frac{\phi(s)}{s^{\frac{\rho n}{n-\alpha}}} \frac{d s}{s} .
$$

By changing variables twice and using that any Young function $\varphi$ satisfy $\varphi(t) \leq$ $\varphi^{\prime}(t) t \leq \varphi(2 t)$, we obtain

$$
\begin{aligned}
\int_{c_{0}}^{\infty} \frac{\psi(v)}{v^{\rho}} \frac{d v}{v} & \lesssim \int_{c_{2}}^{\infty} \frac{u}{\left[\phi^{-1}(u)\right]^{\frac{\rho n}{n-\alpha}}} \frac{d u}{u}=\int_{c_{2}}^{\infty} \frac{u^{1+\frac{\rho \alpha}{n-\alpha}}}{\left[\phi^{-1}(u) u^{\frac{\alpha}{n}}\right]^{\frac{\rho n}{n-\alpha}}} \frac{d u}{u} \lesssim \int_{c_{2}}^{\infty} \frac{u^{1+\frac{\rho \alpha}{n-\alpha}}}{\left[\eta^{-1}(u)\right]^{\frac{\rho n}{n-\alpha}}} \frac{d u}{u} \\
& \leq \int_{c_{3}}^{\infty} \frac{\eta(t)^{1+\frac{\rho \alpha}{n-\alpha}}}{t^{\frac{\rho n}{n-\alpha}}} \frac{\eta(2 t)}{\eta(t)} \frac{d t}{t} \leq \int_{c_{3}}^{\infty} \frac{\eta(2 t)^{1+\frac{\rho \alpha}{n-\alpha}}}{t^{\frac{\rho n}{n-\alpha}}} \frac{d t}{t} \lesssim \int_{c_{4}}^{\infty} \frac{\eta(z)^{1+\frac{\rho \alpha}{n-\alpha}}}{z^{\frac{\rho n}{n-\alpha}}} \frac{d z}{z}
\end{aligned}
$$

Since by hypothesis the right side is finite for some positive constant $c_{4}$, we obtain that $\psi \in B_{\rho}$ for all $\rho>\tau$.

Since $1 \leq \beta<p^{-}$implies $1 \leq \tau<s^{-}$, from (4.2) we have that $w^{\beta} \in A_{\frac{p(\cdot)}{\beta}, \frac{q(\cdot)}{\beta}}$ is equivalent to $w^{\frac{\tau n}{n-\alpha}} \in A_{\frac{s(\cdot)}{\tau}}$. Besides, as in Theorem 2.3,g $\in L_{w^{\frac{n}{n-\alpha}}}^{s(\cdot)}\left(\mathbf{R}^{n}\right)$ with

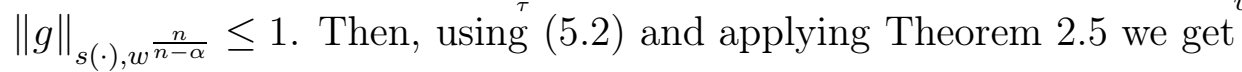

$$
\left\|w M_{\alpha, \eta}(f / w)\right\|_{q(\cdot)} \lesssim\left\|w^{\frac{n}{n-\alpha}} M_{\psi} g\right\|_{s(\cdot)}^{\frac{n-\alpha}{n}} \lesssim\left\|w^{\frac{n}{n-\alpha}} g\right\|_{s(\cdot)}^{\frac{n-\alpha}{n}} \leq 1=\|f\|_{p(\cdot)} .
$$

The next proofs correspond to Wiener's type results.

Proof of Theorem 2.8. Fix $\epsilon>0$. Following the ideas in [10], let us define the auxiliary function $\bar{p}: \mathbf{R}^{n} \rightarrow[1, \infty)$ as

$$
\bar{p}(x)= \begin{cases}\frac{p(x)+1}{2} & \text { if } 1 \leq p(x)<1+\epsilon \\ p(x) & \text { if } p(x) \geq 1+\epsilon\end{cases}
$$

Notice that $p(x) / 2 \leq \bar{p}(x) \leq p(x)$ and $\bar{p}(x) \geq 1$ for every $x \in \mathbf{R}^{n}$. 
On the other hand,

$$
\int_{B} M_{\eta} f(x) w(x) d x \leq w(B)+\int_{1}^{\infty} w\left(\left\{x \in B: M_{\eta} f(x)>t\right\}\right) d t:=w(B)+I .
$$

Using (4.19) and the fact that $\eta$ is a convex function, the integral $I$ can be estimated as follows

$$
\begin{aligned}
I & \lesssim \int_{1}^{\infty} \int_{\{x \in B:|f(x)|>t\}} \eta\left(\frac{|f(x)|}{t}\right) M w(x) d x d t \\
& \lesssim \int_{1}^{\infty} \int_{\{x \in B:|f(x)|>t\}} \eta\left(\left(\frac{|f(x)|}{t}\right)^{\bar{p}(x)}\right) M w(x) d x d t \\
& \lesssim \int_{1}^{\infty} \int_{\{x \in B:|f(x)|>t\}} t^{-\bar{p}(x)} \eta\left(|f(x)|^{\bar{p}(x)}\right) M w(x) d x d t \\
& \lesssim \int_{\{x \in B:|f(x)|>1\}} \eta\left(|f(x)|^{\bar{p}(x)}\right) M w(x)\left(\int_{1}^{|f(x)|} t^{-\bar{p}(x)} d t\right) d x .
\end{aligned}
$$

Let us estimate $\int_{1}^{|f(x)|} t^{-\bar{p}(x)} d t$ for different values of $\bar{p}$. If $\bar{p}(x)=1$, it is clear that $\int_{1}^{|f(x)|} t^{-\bar{p}(x)} d t=\log (|f(x)|) \leq \log (e+|f(x)|)^{q(x)}$ since $q(x)=1$. If $\bar{p}(x) \geq 1+\epsilon$, we have $q(x)=0$ and, therefore,

$$
\int_{1}^{|f(x)|} t^{-\bar{p}(x)} d t=\frac{1-|f(x)|^{1-\bar{p}(x)}}{\bar{p}(x)-1} \leq \frac{1}{\epsilon}=\frac{1}{\epsilon} \log (e+|f(x)|)^{q(x)} .
$$

Now, if $1<\bar{p}(x)<1+\epsilon$, we have $q(x)=\epsilon^{-1}(\epsilon+1-p(x)), 1<\bar{p}(x)<1+\frac{\epsilon}{2}$ and $\bar{p}(x)-1=(p(x)-1) / 2$.

In [10], the authors proved that, for any $a>1$, the function

$$
A(y)=\frac{a^{y}-1}{y} \chi_{(0,1]}(y)+\log a \chi_{\{y=0\}}(y)
$$

is a $\log$-convex function, which means that $\log (A)$ is a convex function. Moreover, given $0<\epsilon \leq 1$, they obtain the following inequality

$$
A(y) \leq \frac{1}{\epsilon} a^{y}(\log a)^{1-\frac{y}{\epsilon}}
$$

for every $0 \leq y \leq \epsilon$. Thus, if $|f(x)|>1$, by taking $a=|f(x)|$ in (5.4) we obtain that

$$
A(y) \leq \frac{2}{\epsilon}|f(x)|^{y} \log (|f(x)|)^{1-\frac{2 y}{\epsilon}}
$$

for every $0 \leq y \leq \frac{\epsilon}{2}$. 
Now, taking $y=\bar{p}(x)-1$, since $1-\frac{2(\bar{p}(x)-1)}{\epsilon}=q(x)$, we obtain that

$$
\begin{aligned}
\int_{1}^{|f(x)|} t^{-\bar{p}(x)} d t & =|f(x)|^{1-\bar{p}(x)} \frac{|f(x)|^{\bar{p}(x)-1}-1}{\bar{p}(x)-1} \leq \frac{|f(x)|^{\bar{p}(x)-1}-1}{\bar{p}(x)-1} \\
& \leq \frac{2}{\epsilon}|f(x)|^{\bar{p}(x)-1} \log (|f(x)|)^{q(x)} \leq C|f(x)|^{(p(x)-1) / 2} \log (e+|f(x)|)^{q(x)} .
\end{aligned}
$$

Finally, if we split the last integral in (5.3) taking into account the three cases above and use that $\eta$ is a convex function, we obtain that

$$
\begin{aligned}
& \int_{B} M_{\eta} f(x) w(x) d x \lesssim \int_{\{|f|>1, \bar{p}=1\}} \eta\left(|f(x)|^{p(x)}\right) \log (e+|f(x)|)^{q(x)} M w(x) d x \\
& \quad+\int_{\{|f|>1, \bar{p} \geq 1+\epsilon\}} \eta\left(|f(x)|^{p(x)}\right) \log (e+|f(x)|)^{q(x)} M w(x) d x \\
& \quad+\int_{\{|f|>1,1<\bar{p}<1+\epsilon\}} \eta\left(|f(x)|^{\frac{p(x)+1}{2}}\right)|f(x)|^{\frac{p(x)-1}{2}} \log (e+|f(x)|)^{q(x)} M w(x) d x \\
& \leq \int_{\{|f|>1\}} \eta\left(|f(x)|^{p(x)}\right) \log (e+|f(x)|)^{q(x)} M w(x) d x .
\end{aligned}
$$

Proof of Theorem 2.9. Since $\eta \in B_{q}$ for every $q>1$, then $\eta(t) \lesssim t^{q}$ for every $t \geq t_{0}$. Without loss of generality we can suppose $t_{0}=1$. We define $\bar{p}$ as in the previous proof. Then, we have

$$
\int_{B} M_{\eta} f(x) w(x) d x \leq w(B)+\int_{\{x \in B:|f(x)|>1\}} M w(x) \int_{1}^{|f(x)|} \eta\left(\frac{|f(x)|}{t}\right) d t d x .
$$

When $\bar{p}(x)=1$, then $q(x)=1$ and by the convexity of $\eta$ we obtain that

$$
\begin{aligned}
\int_{1}^{|f(x)|} \eta\left(\frac{|f(x)|}{t}\right) d t & =\int_{1}^{|f(x)|} \eta(u) \frac{|f(x)|}{u} \frac{d u}{u} \leq|f(x)| \int_{1}^{|f(x)|} \eta^{\prime}(u) \frac{d u}{u} \\
& \leq|f(x)| \eta^{\prime}(|f(x)|) \log (e+|f(x)|) .
\end{aligned}
$$

In the other two cases, since $\bar{p}(x)>1$, we use that $\eta \in B_{\bar{p}(x)}$ :

$$
\int_{1}^{|f(x)|} \eta\left(\frac{|f(x)|}{t}\right) d t \lesssim \int_{1}^{|f(x)|}\left(\frac{|f(x)|}{t}\right)^{\bar{p}(x)} d t=|f(x)|^{\bar{p}(x)} \int_{1}^{|f(x)|} t^{-\bar{p}(x)} d t .
$$

Finally, we estimate the last integral as we did in the proof above, and we use the fact that $\eta^{\prime}(|f(x)|)^{q(x)} \geq 1$ since $\eta^{\prime}(|f(x)|) \geq 1$ and $q(x) \geq 0$.

Proof of Theorem 2.11. Fix $0<\epsilon<1$. We define $r(\cdot)$ and $q(\cdot)$ as in Theorem 1.5 of [10]. For the sake of completeness we include the definition and properties.

Let $R(\cdot)$ be defined by

$$
R(x)=p(x)+(p(x)-1)(p(x)-(1+\epsilon))=(p(x)-1)(p(x)-\epsilon)+1 .
$$

Following [10], and from the fact that $p(\cdot)$ satisfies the log-Hölder condition (1.3) and $p^{+}<\infty$ we have that $p(\cdot)^{2}$ and then, $R(\cdot)$ are also log-Hölder continuous functions.

We now define $r(x)=\min (p(x), R(x))$ which also satisfies the log-Hölder condition (1.3). It is easy to see that when $p(x)=1, R(x)=1$ and when $p(x) \geq 1+\epsilon$, $R(x) \geq p(x)$, so in both cases $r(x)=p(x)$. On the other hand, for $1<p(x)<1+\epsilon$, we have $1<R(x)<p(x)$ which implies $1<r(x)<p(x)$. 
In order to define $q(\cdot)$, let $F=\{x: p(x) \leq 1+\epsilon / 3\}$ and let

$$
r^{*}= \begin{cases}\sup _{x \in F} r(x) & \text { if } F \neq \emptyset \\ 1+\epsilon / 3 & \text { if } F=\emptyset\end{cases}
$$

which is log-Hölder continuous.

We define $\tilde{r}(x)=\min \left(r(x), r^{*}\right)$ and finally

$$
q(x)=\max \left\{\frac{3}{\epsilon}\left(1+\frac{\epsilon}{3}-\frac{p(x)}{\tilde{r}(x)}\right), 0\right\} .
$$

Then, $\tilde{r}(\cdot)$ satisfies condition $(1.3)$, so $q(\cdot)$ is also log-Hölder continuous. Since $\tilde{r}(x) \leq$ $r(x) \leq p(x)$, we have that $0 \leq q(x) \leq 1$. Moreover, when $p(x)=1$, we have $\tilde{r}(x)=1$ so $q(x)=1$. And when $p(x) \geq 1+\epsilon, \frac{p(x)}{\tilde{r}(x)} \geq 1+\frac{\epsilon}{3}$ which gives us $q(x)=0$.

Fix a weight $w$ and a function $f$ as in the hypothesis. Let us split the ball $B$ in the following subsets

$$
\begin{aligned}
& B_{1}=\left\{x \in B: M_{\eta} f(x) \leq 1, p(x)>1+\epsilon / 3\right\} \\
& B_{2}=\left\{x \in B: M_{\eta} f(x)>1, p(x)>1+\epsilon / 3\right\} \\
& B_{3}=\{x \in B: p(x) \leq 1+\epsilon / 3\} .
\end{aligned}
$$

Then

$$
\int_{B} M_{\eta} f(x)^{r(x)} w(x) d x \leq \sum_{i=1}^{3} \int_{B_{i}} M_{\eta} f(x)^{r(x)} w(x) d x .
$$

We estimate each integral separately. For the first one we have

$$
\int_{B_{1}} M_{\eta} f(x)^{r(x)} w(x) d x \leq w\left(B_{1}\right) \leq w(B) .
$$

We will estimate now the last term since it easily follows from Theorem 4.17 with $r(\cdot)=\tilde{r}(\cdot)$ instead of $p(\cdot) \geq r(\cdot)$ and Theorem 2.8 with $\epsilon / 3$ and $p(\cdot) / \tilde{r}(\cdot)$. In fact,

$$
\begin{aligned}
\int_{B_{3}} M_{\eta} f(x)^{r(x)} w(x) d x & \lesssim \int_{B_{3}} S(x)^{\tilde{r}^{-}} w(x)+\int_{B_{3}} M_{\eta}\left(|f(\cdot)|^{\tilde{r}(\cdot)}\right)(x) w(x) d x \\
& \leq w\left(B_{3}\right)+\int_{B} \eta\left(|f(x)|^{\tilde{r}(x) \frac{p(x)}{\tilde{r}(x)}}\right) \log \left(e+|f(x)|^{\tilde{r}(x)}\right)^{q(x)} M w(x) d x \\
& \lesssim w(B)+\int_{\mathbf{R}^{n}} \eta\left(|f(x)|^{p(x)}\right) \log (e+|f(x)|)^{q(x)} M w(x) d x,
\end{aligned}
$$

where the exponent $q(\cdot)$ is given in $(5.5)$.

Finally, to estimate $\int_{B_{2}} M_{\eta} f(x)^{r(x)} w(x) d x$, we need to consider both cases $p^{-}=1$ and $p^{-}>1$. By hypotesis, if $p^{-}>1$, we assume $\eta \in B_{p^{-}}$. Then, since $\eta$ is submupltiplicative, there exist $1<\tau<p^{-}$such that $\eta \in B_{\frac{p^{-}}{\tau}} \subset B_{\frac{p_{B_{2}}}{\tau}}$. On the other hand, if $p^{-}=1, \eta \in B_{q}$ for all $q>1$. Then, since $\frac{p_{B_{2}}^{-}}{1+\epsilon / 6}>\frac{p_{B_{2}}^{-}}{1+\epsilon / 3} \geq 1$, we have $\eta \in B_{\frac{p_{B_{2}}^{-}}{1+\epsilon / 6}}$. Thus, in both cases, there exists a constant $\lambda \in\left(1, p_{B_{2}}^{-}\right)$such that $\eta \in B_{\frac{p_{B_{2}}^{-}}{\lambda}}$.

Hence, from Theorem 4.17 we have that for every $x \in B_{2}$

$$
M_{\eta} f(x)^{p(x)} \lesssim S(x)^{p(x)}+M\left(f(\cdot)^{\frac{p(\cdot)}{\lambda}}\right)(x)^{\lambda},
$$


and thus, from the fact that $r(x) \leq p(x)$

$$
\begin{aligned}
\int_{B_{2}} M_{\eta} f(x)^{r(x)} w(x) d x & \leq \int_{B_{2}} M_{\eta} f(x)^{p(x)} w(x) d x \\
& \lesssim \int_{B_{2}} S(x)^{p^{-}} w(x) d x+\int_{B_{2}} M\left(f(\cdot)^{\frac{p(\cdot)}{\lambda}}\right)(x)^{\lambda} w(x) d x \\
& \leq 2 w\left(B_{2}\right)+\int_{B_{2}}\left[M\left(f(\cdot) \chi_{\{|f|>1\}}\right)^{\frac{p(\cdot)}{\lambda}}\right]^{\lambda}(x) w(x) d x \\
& \lesssim w(B)+\int_{\mathbf{R}^{n}}\left[M\left(f(\cdot) \chi_{\{|f|>1\}}\right)^{\frac{p(\cdot)}{\lambda}}\right]^{\lambda}(x) w(x) d x
\end{aligned}
$$

Since $\lambda>1$, it is well known (see, for example, [20]) that

$$
\int_{\mathbf{R}^{n}}\left[M\left(f(\cdot) \chi_{\{|f|>1\}}\right)^{\frac{p(\cdot)}{\lambda}}\right]^{\lambda}(x) w(x) d x \lesssim \int_{\left\{x \in \mathbf{R}^{n}:|f(x)|>1\right\}}|f(x)|^{p(x)} M w(x) d x .
$$

Thus,

$$
\int_{B_{2}} M_{\eta} f(x)^{r(x)} w(x) d x \lesssim w(B)+\int_{\mathbf{R}^{n}} \eta\left(|f(x)|^{p(x)}\right) \log (e+|f(x)|)^{q(x)} M w(x) d x
$$

since $q(x) \geq 0$.

5.7. Remark. If $\eta \in B_{q}$ for every $q>1$, we can improve the estimate in (5.6) by using Theorem 2.9 in order to get

$\int_{B_{3}} M_{\eta} f(x)^{r(x)} w(x) d x \lesssim w(B)+\int_{\mathbf{R}^{n}}|f(x)|^{p(x)}\left[\eta^{\prime}(|f(x)|) \log (e+|f(x)|)\right]^{q(x)} M w(x) d x$.

Proof of Theorem 2.15. Let us first notice that, from Theorem 4.9 and the hypotesis over $f$, the following inequality holds

$$
M_{\alpha, \eta} f(x)^{\frac{n}{n-\alpha}} \lesssim M_{\psi}\left(|f(\cdot)|^{p(\cdot) / s(\cdot)}\right)(x) .
$$

Then, we can apply Theorem 2.8 for $\psi$ instead of $\eta$ and $s(\cdot)$ instead of $p(\cdot)$ to obtain

$$
\begin{aligned}
\int_{B} M_{\alpha, \eta} f(x)^{\frac{n}{n-\alpha}} w(x) d x & \lesssim \int_{B} M_{\psi}\left(|f(\cdot)|^{p(\cdot) / s(\cdot)}\right)(x) w(x) d x \\
& \lesssim w(B)+\int_{\mathbf{R}^{n}} \psi\left(|f(x)|^{p(x)}\right) \log (e+|f(x)|)^{r(x)} M w(x) d x
\end{aligned}
$$

where $r(x)=\max \left\{\epsilon^{-1}(\epsilon+1-s(x)), 0\right\}$.

\section{References}

[1] Almeida, A., and S. SAmko: Characterization of Riesz and Bessel potentials on variable Lebesgue spaces. - J. Funct. Spaces Appl. 4:2, 2006, 113-144.

[2] Bernardis, A., S. Hartzstein, and G. Pradolini: Weighted inequalities for commutators of fractional integrals on spaces of homogeneous type. - J. Math. Anal. Appl. 322, 2006, 825846.

[3] Bernardis, A. L., M. Lorente, G. Pradolini, and M. S. Riveros: Composition of fractional Orlicz maximal operators and $A_{1}$-weights on spaces of homogeneous type. - Acta Math. Sin. (Engl. Ser.) 26:8, 2010, 1509-1518. 
[4] Bernardis, A. L., M. Lorente, and M. S. Riveros: Weighted inequalities for fractional integral operators with kernel satisfying Hörmander type conditions. - Math. Inequal. Appl. 14:4, 2011, 881-895.

[5] Capone, C., D. Cruz-Uribe, and A. Fiorenza: The fractional maximal operator and fractional integrals on variable $L^{p}$ spaces. - Rev. Mat. Iberoam. 23:3, 2007, 743-770.

[6] Chen, Y., S. Levine, and M. RaO: Variable exponent, linear growth functionals in image restoration. - SIAM J. Appl. Math. 66:4, 2006, 1383-1406.

[7] Coifman, R. R.: Distribution function inequalities for singular integrals. - Proc. Natl. Acad. Sci. USA 69:10, 1972, 2838-2839.

[8] Coifman, R. R., and C. Fefferman: Weighted norm inequalities for maximal functions and singular integrals. - Studia Math. 51, 1974, 241-250.

[9] Cruz-Uribe, D., L. Diening, and P. HÄstö: The maximal operator on weighted variable Lebesgue spaces. - Fract. Calc. Appl. Anal. 14:3, 2011, 361-374.

[10] Cruz-Uribe, D., and A. Fiorenza: Endpoint estimates and weighted norm inequalities for commutators of fractional integrals. - Publ. Mat. 47:1, 2003, 103-131.

[11] Cruz-Uribe, D., and A. Fiorenza: $L \log L$ results for the maximal operator in variable $L^{p}$ spaces. - Trans. Amer. Math. Soc. 361:5, 2009, 2631-2647.

[12] Cruz-Uribe, D., and A. Fiorenza: Introduction to the variable Lebesgue spaces. - Adv. Courses Math. CRM Barcelona (to appear).

[13] Cruz-Uribe, D., A. Fiorenza, J. M. Martell, and C. Pérez: The boudedness of classical operators on variable $L^{p}$ spaces. - Ann. Acad. Sci. Fenn. Math. 31, 2006, 239-264.

[14] Cruz-Uribe, D., A. Fiorenza, and C. J. Neugebauer: The maximal function on variable $L^{p}$ spaces. - Ann. Acad. Sci. Fenn. Math. 28, 2003, 223-238.

[15] Diening, L.: Maximal function on generalized Lebesgue spaces $L^{p(\cdot)}$. - Math. Inequal. Appl. $7: 2,2004,245-254$.

[16] Diening, L.: Maximal function on Musielak-Orlicz spaces and generalized Lebesgue spaces. Bull. Sci. Math. 129:8, 2005, 657-700.

[17] Diening, L., P. Harjulehto, P. Hästö, and M. RŮŽıČKa: Lebesgue and Sobolev spaces with variable exponents. - Lecture Notes in Math. 2017, Springer, 2011.

[18] Diening, L., and M. RŮŽıČKA: Calderón-Zygmund operators on generalized Lebesgue spaces $L^{p(\cdot)}$ and problems related to fluid dynamics. - J. Reine Angew. Math. 563, 2003, 197-220.

[19] Ding, Y., S.Z. Lu, and P. ZhANG: Weak estimates for commutators of fractional integral operators. - Sci. China Math. (Ser. A) 44:7, 2001, 877-888.

[20] Duonndikoetxea, J.: Fourier analysis. - Grad. Stud. Math. 29, 2001.

[21] Gorosito, O., G. Pradolini, and O. Salinas: Boundedness of fractional operators in weighted variable exponent spaces with non doubling measures. - Czechoslovak Math. J. 60:135, 2010, 1007-1023.

[22] Gorosito, O., G. Pradolini, and O. Salinas: Boundedness of the fractional maximal operator on variable exponent Lebesgue spaces: A short proof. - Rev. Un. Mat. Argentina $53: 1,2012,25-27$.

[23] Gorosito, O., G. Pradolini, and O. Salinas: Maximal operators on weighted variable Lebesgue spaces. - Preprint.

[24] Gurka, P., P. Harjulehto, and A. Nekvinda: Bessel potential spaces with variable exponent. - Math. Inequal. Appl. 10:3, 2007, 661-676.

[25] Hedberg, L.-I.: On certain convolution inequalities. - Proc. Amer. Math. Soc. 36, 1972, $505-510$. 
[26] Hu, G., X. SHI, and Q. Zhang: Weighted norm inequalities for the maximal singular integrals operators on spaces of homogeneous type. - J. Math. Anal. Appl. 336, 2007, 1-17.

[27] Kanashiro, A., G. Pradolini, and O. Salinas: Weighted modular estimates for a generalized maximal operator on spaces of homogeneous type. - Collect. Math. 63:2, 2010, 147-164.

[28] Kokilashvili, V., and S. SAmko: Maximal and fractional operators in weighted $L^{p(x)}$ spaces. - Rev. Mat. Iberoam. 20:2, 2004, 493-515.

[29] Kováčı́, O., and J. RÁkosník: On spaces $L^{p(x)}$ and $W^{k, p(x)}$. - Czechoslovak Math. J. 41:4, $1991,592-618$.

[30] Lerner, A.: On modular inequalities in variable $L^{p}$ spaces. - Arch. Math. (Basel) 85:6, 2005, $538-543$.

[31] Lerner, A.: Some remarks on the Hardy-Littlewood maximal function on variable $L^{p}$ spaces. - Math. Z. 251:3, 2005, 509-521.

[32] Lerner, A.: On some questions related to the maximal operator on variable $L^{p}$ spaces. Trans. Amer. Math. Soc. 362:8, 2010, 4229-4242.

[33] Lerner, A., and A. Karlovich: Commutators of singular integrals on generalized $L^{p}$ spaces with variable exponent. - Publ. Mat. 49:1, 2005, 111-125.

[34] Lorente, M., J. M. Martell, M. S. Riveros, and A. De la Torre: Generalized Hörmander's conditions, commutators and weights. - J. Math. Anal. Appl. 342:2, 2008, 1399-1425.

[35] Lorente, M., M. S. Riveros, and A. De la Torre: Weighted estimates for singular integral operators satisfying Hörmander's conditions of Young type. - J. Fourier Anal. Appl. 11:5, 2005, 497-509.

[36] Muckenhoupt, B., and R. Wheeden: Weighted norm inequalities for fractional integral. Trans. Amer. Math. Soc. 192, 1974, 261-274.

[37] Musielak, J.: Orlicz spaces and Modular spaces. - Lecture Notes in Math. 1034, SpringerVerlag, 1983.

[38] Nekvinda, A.: Hardy-Littlewood maximal operator on $L^{p(x)}\left(\mathbf{R}^{n}\right)$. - Math. Inequal. Appl. $7: 2,2004,255-265$.

[39] Nekvinda, A.: A note on maximal operator on $\ell^{\left\{p_{n}\right\}}$ and $L^{p(x)}(\mathbf{R})$. - J. Funct. Spaces Appl. $5: 1,2007,49-88$.

[40] Nekvinda, A.: Maximal operator on variable Lebesgue spaces for almost monotone radial exponent. - J. Math. Anal. Appl. 337:2, 2008, 1345-1365.

[41] PÉRez, C.: Weighted norm inequalities for singular integral operators. - J. Lond. Math. Soc. 49, 1994, 296-308.

[42] PÉrez, C.: Endpoint estimates for commutators of singular integral operators. - J. Funct. Anal. 128, 1995, 163-185.

[43] PÉrez, C.: Sharp estimates for commutators of singular integrals via iterations of the HardyLittlewood maximal function. - J. Fourier Anal. Appl. 3:6, 1997, 743-756.

[44] PÉrez, C., and G. Pradolini: Sharp weighted endpoint estimates for commutators of singular integral operators. - Michigan Math. J. 49, 2001, 23-37.

[45] PICK, L., and M. RŮŽIČKA: An example of a space $L^{p(x)}$ on which the Hardy-Littlewood maximal operator is not bounded. - Expo. Math. 19:4, 2001, 369-371.

[46] Rao, M., and Z. Ren: Theory of Orlicz spaces. - Marcel Dekker, New York, 1991.

[47] RŮŽIČKA, M.: Electrorheological fluids: Modeling and mathematical theory. - Lecture Notes in Math. 1748, Springer, 2000.

[48] Wiener, N.: The ergodic theorem. - Duke Math. J. 5, 1939, 1-18. 